\title{
Morse theory for manifolds with boundary
}

\author{
MACIEJ BORODZIK \\ ANDRÁS NÉMETHI \\ ANDREW RANICKI
}

\begin{abstract}
We develop Morse theory for manifolds with boundary. Beside standard and expected facts like the handle cancellation theorem and the Morse lemma for manifolds with boundary, we prove that under suitable connectedness assumptions a critical point in the interior of a Morse function can be moved to the boundary, where it splits into a pair of boundary critical points. As an application, we prove that every cobordism of connected manifolds with boundary splits as a union of left product cobordisms and right product cobordisms.
\end{abstract}

57R19; 58E05, 58A05

\section{Introduction}

For some time now, Morse theory has been a very fruitful tool in the topology of manifolds. One of its milestones was the $h$-cobordism theorem of Smale [17], and its Morse-theoretic exposition by Milnor [11; 12]. Recently, Morse theory has become even more popular, for two reasons: its connections with Floer homology (see eg Salamon [15], Witten [18], Nicolaescu [13] and Kronheimer and Mrowka [9]) and the stratified Morse theory developed by Goresky and MacPherson [6]. In the last 20 years Morse theory has also had an enormous impact on the singularity theory of complex algebraic and analytic varieties.

Morse theory for manifolds with boundary was studied in the seventies by Braess [5], Jankowski and Rubinsztein [8] and Hajduk [7]. Recently it was developed by Kronheimer and Mrowka [9]. Since then the theory has experienced very fast development, as witnessed by the papers of Bloom [2] and Laudenbach [10]. Our paper is another contribution.

In this paper we prove some new results in Morse theory for manifolds with boundary. Besides some standard and expected results, like the boundary handle cancellation theorem (Theorem 5-1) and the topological description of passing critical points on the boundary (using the notions of right and left half-handles introduced in Section 2) 
we describe another phenomenon; see Theorem 3-1. An interior critical point can be moved to the boundary and there split into two boundary critical points. A related result was stated in Hajduk [7, Theorem 5]; we provide a rigorous proof under much weaker assumptions.

In particular, if we have a cobordism of manifolds with boundary, then under a natural topological assumption we can find a Morse function which has only boundary critical points. We use this result to prove a structure theorem for connected cobordisms of connected manifolds with connected nonempty boundary: such a cobordism splits as a union of left and right product cobordisms. This is a topological counterpart to the algebraic splitting of cobordisms obtained by the authors in [3, Main theorem 1]: an algebraic splitting of the chain complex cobordism of a geometric cobordism can be realized topologically by a geometric splitting. This algebraic splitting is used to study the algebraic properties of the Seifert matrices of isotopic nonspherical $(2 n-1)-$ dimensional links in $S^{2 n+1}$. This will provide the algebraic background to our proof that the semicontinuity of mod 2 spectra of hypersurface singularities is a purely topological phenomenon (see the authors' [4], especially the paragraph before proof of Theorem 2.1.8).

The structure of the paper is as follows. After preliminaries in Section 1.1 we study in Section 2 the changes in the topology of the level sets when crossing a boundary critical point. Theorem 2-27 is the main result of this section: passing a boundary stable (unstable) critical point produces a left (right) half-handle attachment. In Section 3 we prove Theorem 3-1, which moves interior critical points to the boundary. This is the first main result of this article. Then we pass to some more standard results, namely rearrangements of critical points in Section 4. We finish the section with our most important - up to now - application, Theorem 4-18, about the splitting of a cobordism into left product and right product cobordisms. Finally, in Section 5 we discuss the possibility of canceling a pair of critical points. We include this part for completeness, the main result of this section was proved in Hajduk [7, Theorem 1].

Acknowledgements The first author wishes to thank Rényi Institute for its hospitality, the first two authors are grateful for Edinburgh Mathematical Society for a travel grant to Edinburgh and Glasgow in March 2012. The authors thank András Juhász, Mark Powell and András Stipsicz for fruitful discussions and Rob Kirby for drawing their attention to Kronheimer and Mrowka [9].

The first author is supported by Polish MNiSzW Grant number N201 397937. The second author is partially supported by OTKA Grant K100796. 


\subsection{Notes on gradient vector fields}

To fix notation, let us recall what a cobordism of manifolds with boundary is.

Definition 1-1 Let $\Sigma_{0}$ and $\Sigma_{1}$ be compact oriented, $n$-dimensional manifolds with nonempty boundaries $M_{0}$ and $M_{1}$. We shall say that $(\Omega, Y)$ is a cobordism between $\left(\Sigma_{0}, M_{0}\right)$ and $\left(\Sigma_{1}, M_{1}\right)$ if $\Omega$ is a compact oriented $(n+1)$-dimensional manifold with boundary $\partial \Omega=Y \cup \Sigma_{0} \cup \Sigma_{1}$, where $Y$ is nonempty, $\Sigma_{0} \cap \Sigma_{1}=\varnothing$, and $Y \cap \Sigma_{0}=M_{0}$, $Y \cap \Sigma_{1}=M_{1}$.

Remark 1-2 Strictly speaking, $\Omega$ is a manifold with corners, so around a point $x \in M_{0} \cup M_{1}$ it is locally modeled on $\mathbb{R}^{n-1} \times \mathbb{R}_{\geqslant 0}^{2}$. Accordingly, sometimes we write that $\Sigma_{0}, \Sigma_{1}$ and $Y$, as manifolds with boundary, have tubular neighborhoods in $\Omega$ of the form $\Sigma_{0} \times[0,1), \Sigma_{1} \times[0,1)$, or $Y \times[0,1)$, respectively. Nevertheless, in most cases it is safe (and more convenient) to assume that $\Omega$ is a manifold with boundary, ie that the corners are smoothed along $M_{0}$ and $M_{1}$. Whenever possible we make this simplification in order to avoid unnecessary technicalities.

Example 1-3 Given a manifold with boundary $(\Sigma, M)$, we call $(\Sigma, M) \times[0,1]$ a trivial cobordism, with $\Omega=\Sigma \times[0,1], Y=M \times[0,1], \Sigma_{i}=\Sigma \times\{i\}, M_{i}=M \times\{i\}$ for $i=0,1$.

We recall the notion of a Morse function (in [7] they are called $m$-functions). For this it is convenient to fix a Riemannian metric $g$ on $\Omega$.

Definition 1-4 Let $F: \Omega \rightarrow[0,1]$ be a smooth function. A critical point $z$ of $F$ is called Morse if the Hessian of $F$ at $z$ is nondegenerate. The function $F: \Omega \rightarrow[0,1]$ is called a Morse function on the cobordism $(\Omega, Y)$ if $F\left(\Sigma_{0}\right)=0, F\left(\Sigma_{1}\right)=1, F$ has only Morse critical points, the critical points are not situated on $\Sigma_{0} \cup \Sigma_{1}$, and $\nabla F$ is everywhere tangent to $Y$.

There are two ways of doing Morse theory on manifolds. One can either consider the gradient flow of $\nabla F$ associated with $F$ and the Riemannian metric (in the Floer theory, one often uses $-\nabla F$ ), or the so-called gradient-like vector field.

Definition 1-5 (See Milnor [12, Definition 3.1]) Let $F$ be a Morse function on a cobordism $(\Omega, Y)$. Let $\xi$ be a vector field on $\Omega$. We shall say that $\xi$ is gradient-like with respect to $F$ if the following conditions are satisfied: 
(a) $\xi \cdot F>0$ away from the set of critical points of $F$.

(b) If $p$ is a critical point of $F$ of index $k$, then there exist local coordinates $x_{1}, \ldots, x_{n+1}$ in a neighborhood of $p$, such that

$$
F\left(x_{1}, \ldots, x_{n+1}\right)=F(p)-\left(x_{1}^{2}+\cdots+x_{k}^{2}\right)+\left(x_{k+1}^{2}+\cdots+x_{n+1}^{2}\right)
$$

and

$$
\xi=\left(-x_{1}, \ldots,-x_{k}, x_{k+1}, \ldots, x_{n+1}\right) \text { in } U
$$

$\left(\mathrm{b}^{\prime}\right)$ Also, if $p$ is a boundary critical point, then the above coordinate system can be chosen so that $Y=\left\{x_{j}=0\right\}$ and $U=\left\{x_{j} \geqslant 0\right\}$ for some $j \in\{1, \ldots, n+1\}$.

(c) $\xi$ is everywhere tangent to $Y$.

The conditions (a) and (b) are the same as in the classical case. Condition ( $\left.b^{\prime}\right)$ is an analogue of condition (b) in the boundary case; cf Lemma 2-6.

Smale [16] noticed that for any gradient-like vector field $\xi$ for a function $F$ there exists a Riemannian metric such that $\xi=\nabla F$ in that metric. The situation is identical in the boundary case. This is stated explicitly in the following lemma, whose proof is straightforward and will be omitted.

Lemma 1-6 Let $U$ be a paracompact $k$-dimensional manifold and $F: U \rightarrow \mathbb{R}$ a Morse function without critical points. Assume that $\xi$ is a gradient-like vector field on $U$. Then there exists a metric $g$ on $U$ such that $\xi=\nabla F$ in that metric. A similar statement holds if $U$ has boundary and $\xi$ is everywhere tangent to the boundary.

Hence the two approaches — by gradients and gradient-like vector fields - are equivalent. We shall need both of them. In Section 3 we use gradients of functions and a specific choice of a metric, which make the arguments slightly simpler. In Section 5 we follow Milnor [12] very closely; as he uses gradient-like vector fields, we use them as well.

The next result shows that the condition from Definition 1-4 that $\nabla F$ is everywhere tangent to $Y$ can be relaxed. We shall use this result in Proposition 4-1.

Lemma 1-7 Let $\Omega$ be a compact Riemannian manifold of dimension $n+1$ and let $Y \subset \partial \Omega$ be compact as well. Let $g$ denote the metric. Suppose that there exists a function $F: \Omega \rightarrow \mathbb{R}$, and a relative open subset $U \subset Y$ such that $\nabla F$ is tangent to $Y$ at each point $y \in U$. Suppose furthermore that for any $y \in Y \backslash U$ we have

$$
T_{y} Y \not \subset \operatorname{ker} d F \text {. }
$$


Then, for any open neighborhood $W \subset \Omega$ of $Y \backslash U$, there exist a metric $h$ on $\Omega$, agreeing with $g$ away from $W$, such that $\nabla_{h} F$ (the gradient in the new metric) is everywhere tangent to $Y$.

Proof Let us fix a point $y \in Y \backslash U$ and consider a small open neighborhood $V_{y}$ of $y$ in $W$, in which we choose local coordinates $x_{1}, \ldots, x_{n+1}$ such that $Y \cap V_{y}=\left\{x_{n+1}=0\right\}$ and $V_{y} \subset\left\{x_{n+1} \geqslant 0\right\}$. In these coordinates we have $d F=\sum_{i=1}^{n+1} f_{i}(x) d x_{i}$ for some smooth functions $f_{1}, \ldots, f_{n+1}$. By (1-8), for each $x \in V_{y}$, there exists $i \leqslant n$ such that $f_{i}(x) \neq 0$. Shrinking $V_{y}$ if needed, we may assume that the index $i$ is the same for each $x \in V_{y}$. In the following we suppose that $i=1$, that is for every $x \in V_{y}$ we have $\pm f_{1}(x)>0$ and the sign \pm is the same for every $x$. Let us choose a symmetric positive-definite matrix $A_{y}=\left\{a_{i j}(x)\right\}_{i, j=1}^{n+1}$ so that $a_{11}= \pm f_{1}(x)$ and for $i>1$, $a_{1 i}=a_{i 1}=f_{i}(x) . A_{y}$ defines a metric $h_{y}$ on $V_{y}$ such that $\nabla_{h_{y}} F=( \pm 1,0, \ldots, 0) \subset$ $T Y$ in that metric.

Now let us choose an open subset $V$ of $\Omega \backslash(Y \backslash U)$ such that $V \cup \bigcup_{y \in Y \backslash U} V_{y}$ is a covering of $\Omega$. Let $\left\{\phi_{V}\right\} \cup\left\{\phi_{y}\right\}_{y \in Y \backslash U}$ be a partition of unity subordinate to this covering. Define

$$
h=\phi_{V} \cdot g+\sum_{y \in Y \backslash U} \phi_{y} h_{y} .
$$

Then $h$ is a metric, which agrees with $g$ away from $W$. Moreover, as for each metric $h_{y}$, and $x \in V_{y} \cap Y$ we have $\nabla_{h_{y}} F(x) \in T_{x} Y$ by construction, the same holds for a convex linear combination of metrics.

\section{Boundary stable and unstable critical points}

Most of the results of this section appeared previously in $[5 ; 8 ; 7]$. We provide them for completeness of exposition and for the convenience of the reader. In what follows, we use notation and terminology of [9].

\subsection{Morse function for manifolds with boundary}

The first question concerns the existence of Morse functions. While the condition that the function has only critical points of Morse type is open and dense, it requires a little argument to show that there are many functions generic in the interior such that their gradient, when restricted to $Y$, is tangent to $Y$.

Lemma 2-1 Morse functions exist. In fact, for any Morse function $f: Y \rightarrow[0,1]$ with $f\left(M_{0}\right)=0, f\left(M_{1}\right)=1$ there exists a Morse function $F: \Omega \rightarrow[0,1]$ whose restriction to $Y$ is $f$. 
Proof Let $f: Y \rightarrow[0,1]$ be a Morse function on the boundary, such that $f\left(M_{0}\right)=0$ and $f\left(M_{1}\right)=1$. We want to extend $f$ to a Morse function on $\Omega$.

First, let us choose a small tubular neighborhood $U$ of $Y$ and a diffeomorphism $U \cong Y \times[0, \varepsilon)$ for some $\varepsilon>0$. Let $\widetilde{F}: U \rightarrow[0,1]$ be given by the formula

$$
U \cong Y \times[0, \varepsilon) \ni(x, t) \rightarrow \widetilde{F}(x, t)=f(x)-f(x)(1-f(x)) t^{2} .
$$

The factor $f(x)(1-f(x))$ ensures that $\widetilde{F}$ attains values in the interval $[0,1]$ and $\widetilde{F}^{-1}(i) \subset \Sigma_{i}$ for $i \in\{0,1\}$. It is obvious that there exists a smooth function $F: \Omega \rightarrow$ $[0,1]$, which agrees on $Y \times[0, \varepsilon / 2)$ with $\widetilde{F}$, and it satisfies the Morse condition on the whole of $\Omega$. The gradient $\nabla F$ is everywhere tangent to $Y$.

Remark 2-3 The above construction yields a function with the property that all its boundary critical points are boundary stable (see Definition 2-4 below). This is due to the choice of sign -1 in front of $f(x)(1-f(x)) t^{2}$ in (2-2). If we change the sign to +1 , we obtain a function with all boundary critical points boundary unstable.

We fix a Morse function $F: \Omega \rightarrow[0,1]$ and we start to analyze its critical points. Let $z$ be such a point. If $z \in \Omega \backslash Y$, we shall call it an interior critical point. If $z \in Y$, it will be called a boundary critical point. There are two types of boundary critical points.

Definition 2-4 Let $z$ be a boundary critical point. We shall call it boundary stable, if the tangent space to the unstable manifold of $z$ lies entirely in $T_{z} Y$, otherwise it is called boundary unstable.
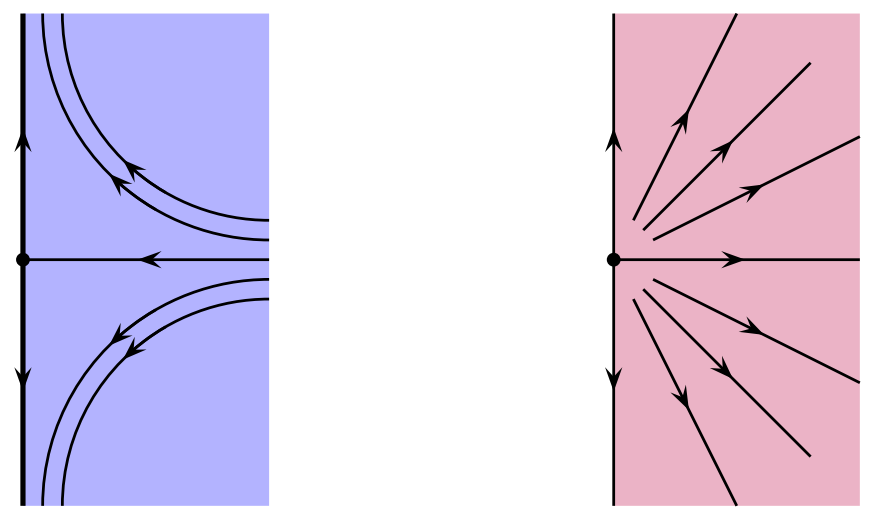

Figure 1: Boundary stable (on the left) and unstable critical points.

The index of the boundary critical point $z$ is defined as the dimension of the stable manifold $W_{z}^{s}$. If $z$ is boundary unstable, this is the same as the index of $z$ regarded 
as a critical point of the restriction $f$ of $F$ on $Y$. If $z$ is boundary stable, we have $\operatorname{ind}_{F} z=\operatorname{ind}_{f} z+1$. In particular, there are no boundary stable critical point with index 0 , nor boundary unstable critical points of index $n+1$.

Remark 2-5 We point out that we use the flow of $\nabla F$ and not of $-\nabla F$ as Kronheimer and Mrowka [9] do, hence our definitions and formulae are slightly different from theirs.

We finish this subsection with three standard results.

Lemma 2-6 (Boundary Morse lemma) Assume that $F$ has a critical point $z \in Y$ such that the Hessian $D^{2} F(z)$ at $z$ is nondegenerate, and $\nabla F$ is everywhere tangent to $Y$. Then there are local coordinates $\left(x_{1}, \ldots, x_{n+1}\right)$ in an open neighborhood $U \ni z$ such that $U=\left\{x_{1}^{2}+\cdots+x_{n+1}^{2} \leqslant \varepsilon^{2}\right\} \cap\left\{x_{1} \geqslant 0\right\}$ and $U \cap Y=\left\{x_{1}=0\right\}$ for some $\varepsilon>0$, and $F$ in these coordinates has the form $\pm x_{1}^{2} \pm x_{2}^{2} \pm \cdots \pm x_{n+1}^{2}+F(z)$.

Proof We choose a coordinate system $y_{1}, \ldots, y_{n+1}$ in a neighborhood $U \subset \Omega$ of $z$ such that $z=(0, \ldots, 0), Y=\left\{y_{1}=0\right\}, U=\left\{y_{1} \geqslant 0\right\}$, and the vector field $\frac{\partial}{\partial y_{1}}$ is orthogonal to $Y$. We may and will assume $F(z)=0$. The tangency of $\nabla F$ to $Y$ implies that at each point of $Y$,

$$
\frac{\partial F}{\partial y_{1}}\left(0, y_{2}, \ldots, y_{n+1}\right)=0 \text {. }
$$

The Hadamard lemma applied to $F$ gives smooth functions $K_{1}, \ldots, K_{n+1}$ such that

$$
F=y_{1} K_{1}\left(y_{1}, \ldots, y_{n+1}\right)+\sum_{j=2}^{n+1} y_{j} K_{j}\left(y_{1}, y_{2}, \ldots, y_{n+1}\right) \text {. }
$$

We can assume that for $j>1, K_{j}$ does not depend on $y_{1}$. Indeed, if it does, we write (again using the Hadamard lemma)

$$
K_{j}\left(y_{1}, \ldots, y_{n+1}\right)=K_{j}\left(0, y_{2}, \ldots, y_{n+1}\right)+y_{1} L_{1 j}\left(y_{1}, \ldots, y_{n+1}\right)
$$

for smooth functions $L_{12}, \ldots, L_{1, n+1}$, and then replace $K_{j}$ by $K_{j}\left(0, y_{2}, \ldots, y_{n+1}\right)$ and $K_{1}$ by $K_{1}+\sum y_{j} L_{1 j}$. Condition (2-7) implies now that $K_{1}\left(0, y_{2}, \ldots, y_{n+1}\right)=0$, hence

$$
K_{1}\left(y_{1}, \ldots, y_{n+1}\right)=y_{1} H_{11}\left(y_{1}, \ldots, y_{n+1}\right)
$$

for some function $H_{11}$. By the Hadamard lemma applied to $K_{2}, \ldots, K_{n+1}$ we infer that there exist functions $H_{j k}$ for $j, k=2, \ldots, n+1$ such that

$$
F=y_{1}^{2} H_{11}\left(y_{1}, \ldots, y_{n+1}\right)+\sum_{j, k=2}^{n} y_{j} y_{k} H_{j k}\left(y_{2}, \ldots, y_{n+1}\right) \text {. }
$$


Notice that the functions $H_{11}$ and $H_{j k}$ for $j, k=2, \ldots, n+1$ evaluated at $z$ correspond to the second derivatives of $F$ at $z$. The nondegeneracy of $D^{2} F(z)$ implies that $H_{11}(z) \neq 0$; by continuity $H_{11}$ does not vanish in a neighborhood of $z$. After replacing $y_{1} \sqrt{ \pm H_{11}}$ by $x_{1}$, we can assume that $H_{11}= \pm 1$. Finally, the sum in (2-9) can be written as $\sum_{j \geqslant 2} \epsilon_{j} x_{j}^{2}\left(\epsilon_{j}= \pm 1\right)$ by the classical Morse lemma [11, Lemma 2.2].

The next result is completely standard by now.

Lemma 2-10 Assume that $F$ is a Morse function on a cobordism $(\Omega, Y)$ between $\left(\Sigma_{0}, M_{0}\right)$ and $\left(\Sigma_{1}, M_{1}\right)$. If $F$ has no critical points, then $(\Omega, Y) \cong\left(\Sigma_{0}, M_{0}\right) \times[0,1]$. Furthermore, we can choose the diffeomorphism to map the level set $F^{-1}(t)$ to the set $\Sigma_{0} \times\{t\}$.

Proof The proof is identical to the classical case; see eg [12, Theorem 3.4].

\subsection{Half-handles}

For any $k$ we consider the $k$-dimensional disk $D^{k}=\left\{x_{1}^{2}+\cdots+x_{k}^{2} \leqslant 1\right\}$. In the classical theory, an $n$-dimensional handle of index $k$ is the $n$-dimensional manifold $H=D^{k} \times D^{n-k}$ with boundary

$$
\partial H=\left(\partial D^{k} \times D^{n-k}\right) \cup\left(D^{k} \times \partial D^{n-k}\right)=B_{0} \cup B_{0}^{\prime} .
$$

Given an $n$-manifold with boundary $(\Sigma, \partial \Sigma)$ and a distinguished embedding $\phi: B_{0} \rightarrow$ $\partial \Sigma$, the effect of a classical handle attachment is the $n$-dimensional manifold with boundary

$$
\left(\Sigma^{\prime}, \partial \Sigma^{\prime}\right)=\left(\Sigma \cup H,\left(\partial \Sigma \backslash B_{0}\right) \cup B_{0}^{\prime}\right),
$$

where we glue along $\phi\left(B_{0}\right)$ identified with $B_{0}$. The boundary $\partial \Sigma^{\prime}$ is the effect of surgery on $\phi\left(B_{0}\right) \subset \partial \Sigma$. We now extend this construction to relative cobordisms of manifolds with boundary, using "half-handles". Since our ambient space $\Omega$ is $(n+1)-$ dimensional, $(n+1)$ is the dimension of the handles, and they induce $n$-dimensional handle attachments on $Y$.

In order to do this, for any $k \geqslant 1$ we distinguish the following subsets of $D^{k}$ : the "half-disk" $D_{+}^{k}:=D^{k} \cap\left\{x_{1} \geqslant 0\right\}$, and its boundary subsets $S_{+}^{k-1}:=\partial D^{k} \cap\left\{x_{1} \geqslant 0\right\}$, $S_{0}^{k-2}:=\partial D^{k} \cap\left\{x_{1}=0\right\}$ and $D_{0}^{k-1}:=D^{k} \cap\left\{x_{1}=0\right\}$. Clearly, $S_{0}^{k-2}$ is a boundary of the two $(k-1)$-disks $S_{+}^{k-1}$ and $D_{0}^{k-1}$; see Figure 2. We will call $x_{1}$ the cutting coordinate. 
Definition 2-11 Let $0 \leqslant k \leqslant n$. An $(n+1)$-dimensional right half-handle of index $k$ is the $(n+1)$-dimensional manifold $H_{\text {right }}=D^{k} \times D_{+}^{n+1-k}$, with boundary subdivided into three pieces $\partial H_{\text {right }}=B \cup C \cup N$, where

$$
B:=\partial D^{k} \times D_{+}^{n+1-k}, \quad C:=D^{k} \times D_{0}^{n-k}, \quad N:=D^{k} \times S_{+}^{n-k} .
$$

One also has the intersections

$$
B_{0}:=C \cap B=\partial D^{k} \times D_{0}^{n-k}, \quad N_{0}:=C \cap N=D^{k} \times S_{0}^{n-k-1} .
$$

Hence the handle $H$ is cut along $C$ into two pieces, one of them is the half-handle $H_{\text {right }}$. Note that $\left(C, B_{0}\right)$ is a $n$-dimensional handle of index $k$. See Figure 3 for an example of a right half-handle.

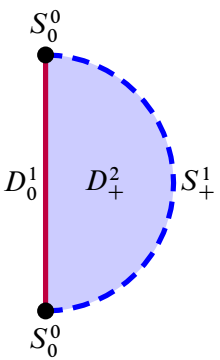

Figure 2: Various parts of a "half-disk" explaning the notation introduced before Definition 2-11.

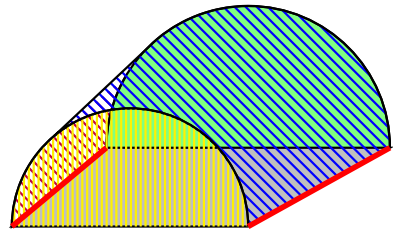

Figure 3: A right half-handle of index 1. The two half-disks form $B$, while $C$ is the bottom rectangle.

Symmetrically, we define the left half-handles by cutting the handle $H$ along the left-component disk $D^{k}$; see Figure 4.

Definition 2-12 Fix $k$ with $1 \leqslant k \leqslant n+1$. An $(n+1)$-dimensional left half-handle of index $k$ is the $(n+1)$-dimensional disk $H_{\text {left }}:=D_{+}^{k} \times D^{n+1-k}$ with boundary subdivided into three pieces $\partial H_{\text {left }}=B \cup C \cup N$, where

$$
B:=S_{+}^{k-1} \times D^{n+1-k}, \quad C:=D_{0}^{k-1} \times D^{n+1-k}, \quad N:=D_{+}^{k} \times \partial D^{n+1-k} .
$$

We also set $B_{0}:=C \cap B=S_{0}^{k-2} \times D^{n+1-k}$ and $N_{0}:=N \cap C=D_{0}^{k-1} \times \partial D^{n+1-k}$. 

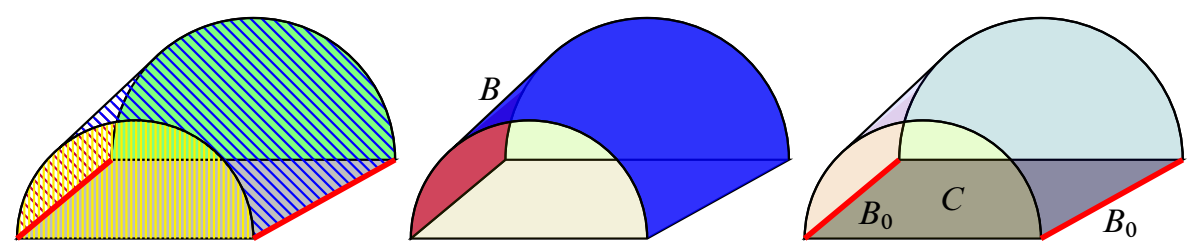

Figure 4: A left half-handle of dimension 3 and index $k=2$. The two lines are $B_{0}$, the bottom rectangle is $C . B$ is the surface between the two half circles in the picture.

Remark 2-13 The right half-handle and left-half handle are abstractly diffeomorphic to an $(n+1)$-dimensional disk. The right half-handle and left-half handle are each abstractly diffeomorphic to an $(n+1)$-dimensional disk. The difference is that the boundary is split into several components and this splitting is different for right halfhandles and left half-handles.

A half-handle will from now on refer to either a right half-handle or left half-handle. We pass to half-handle attachments. We will attach a half-handle along $B$. The definitions of the right half-handle attachment and the left half-handle attachment are formally very similar, but there are significant differences in the properties of the two operations.

Definition 2-14 Let $\left(\Omega, Y ; \Sigma_{0}, M_{0}, \Sigma_{1}, M_{1}\right)$ be an $(n+1)$-dimensional relative cobordism. Given an embedding $\Phi:\left(B, B_{0}\right) \hookrightarrow\left(\Sigma_{1}, M_{1}\right)$ define the relative cobor$\operatorname{dism}\left(\Omega^{\prime}, Y^{\prime} ; \Sigma_{0}, M_{0}, \Sigma_{1}^{\prime}, M_{1}^{\prime}\right)$ obtained from $\left(\Omega, Y ; \Sigma_{0}, M_{0}, \Sigma_{1}, M_{1}\right)$ by attaching $a$ (right or left) half-handle of index $k$ by

$$
\begin{aligned}
\Omega^{\prime} & =\Omega \cup_{B} H, & Y^{\prime} & =Y \cup_{B_{0}} C, \\
\Sigma_{1}^{\prime} & =\left(\Sigma_{1} \backslash B\right) \cup N, & M_{1}^{\prime} & =\left(M_{1} \backslash B_{0}\right) \cup N_{0} .
\end{aligned}
$$

See Figure 5 and Figure 6 for right, respectively left half-handle attachments.

We point out that in the case of the right half-handle attachment, any embedding of $B_{0}$ into $M_{1}$ determines (up to an isotopy) an embedding of pairs $\left(B, B_{0}\right) \hookrightarrow\left(\Sigma_{1}, M_{1}\right)$. Indeed, as $\left(B, B_{0}\right)=\partial D^{k} \times\left(D_{+}^{n+1-k}, D_{0}^{n-k}\right)$, a map $\phi: B_{0} \hookrightarrow M_{1}$ extends to a map $\Phi: B \hookrightarrow \Sigma_{1}$ in a collar neighborhood of $M_{1}$ in $\Sigma_{1}$. (This is not the case in the left half-handle attachment.)

In particular, in the case of right attachments, we specify only the embedding $B_{0} \hookrightarrow M_{1}$.

Example 2-15 (a) The right half-handle attachment of index 0 is the disconnected sum $\Omega \sqcup D_{+}^{n+1}$ with boundary $\partial D_{+}^{n+1}=S_{+}^{n} \cup D_{0}^{n}$. We think of the first disk $S_{+}^{n}$ as a part of $\Sigma_{1}^{\prime}$, while the second disk as a part of $Y^{\prime}$, and $M_{1}^{\prime}=M_{1} \cup S_{0}^{n-1}$. 


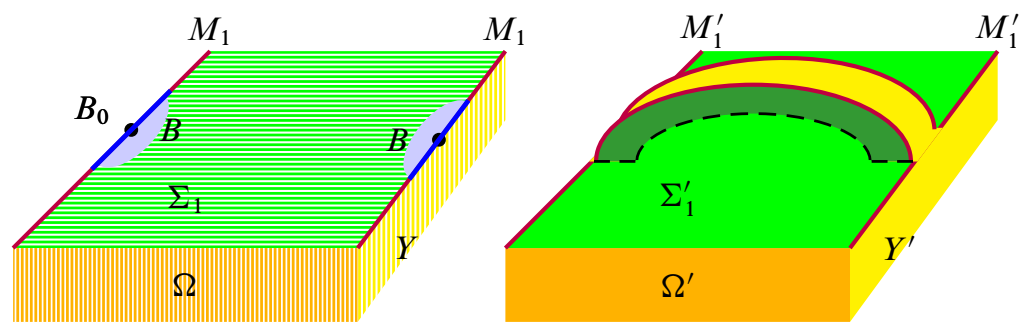

Figure 5: Right half-handle attachment. Here $k=1, n=2$. On the right, the two black points represent a sphere $S^{0}$ with a neighborhood $B_{0}$ in $M_{1}$ and $B$ in $\Sigma_{1}$. In the picture on the right the dark green colored part of the handle belongs to $\Sigma_{1}$, the dashed lines belong to $\Sigma_{1}$ and are drawn only to make the picture look more "three-dimensional".

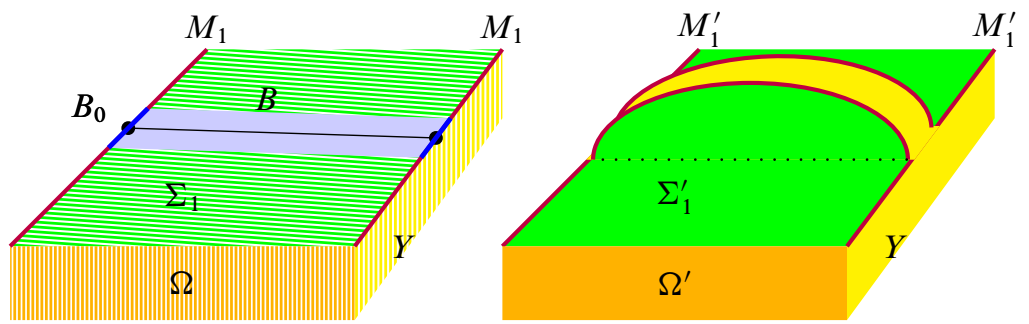

Figure 6: Left half-handle attachment with $k=2$ and $n=2$. This time the sphere on the left (denoted by two points) bounds a disk in $\Sigma_{1}$.

(b) We describe the left half-handle attachment for $k=1$. In this case $B_{0}$ is empty. If we are given an embedding of $B \cong\{1\} \times D^{n}$ into $\Sigma_{1} \backslash M_{1}$, we glue $[0,1] \times D^{n}$ to $\Omega$ along $B$. Then we set $Y^{\prime}=Y \sqcup\{0\} \times D^{n}, \Sigma_{1}^{\prime}=\left(\Sigma_{1} \backslash B\right) \cup[0,1] \times \partial B$ and $M^{\prime}=M \sqcup\{0\} \times \partial B$.

Example 2-16 There is another way of looking at left half-handle attachments. Suppose we are given a model of $\Omega$ (in Figure 7) made of clay. The height function is the Morse function $F$. On the top of $\Omega$, that is on $\Sigma_{1}$, we specify an arc $\gamma$ with boundary in $M_{1}$ (the arc is a 1 -dimensional disk, that is, in our situation $k=1+1=2$ ). We press down slightly a tubular neighborhood of the arc as on the right side of Figure 7. The resulting manifold is a result of a left half-handle attachment of index 2 . Notice that for index-1 left half-handle attachment we should have specified a disk inside $\Sigma_{1}$ (with boundary disjoint from $M_{1}$ ).

Remark 2-17 In the next subsection we shall see that crossing a boundary stable critical point corresponds to a left half-handle attachment, while a boundary unstable 

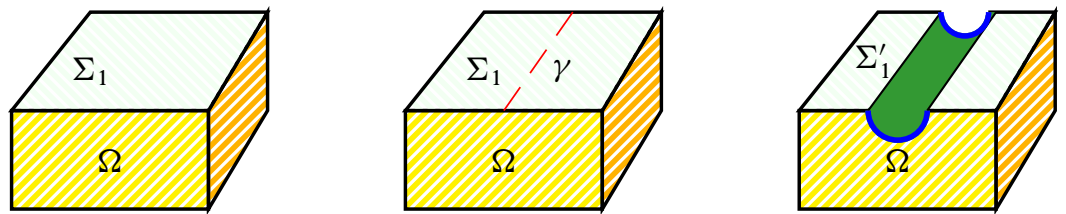

Figure 7: A "clay" variant of a left half-handle attachment as explained in Example 2-16. There are only small differences between this picture and Figure 6 . Here the handle is "pushed down inside $\Omega$ ", in the formal definition it is glued on top of $\Sigma_{1}$.

critical point corresponds to a right half-handle attachment. Theorem 3-1 can be interpreted informally as splitting a handle into a right half-handle and a left halfhandle. This also motivates the name "half-handle".

\subsection{Elementary properties of half-handle attachments}

The following results are trivial consequences of the definitions.

Lemma 2-18 Let $\Omega^{\prime}$ be the result of a right half-handle attachment to $\Omega$ along $\left(B, B_{0}\right) \hookrightarrow\left(\Sigma_{1}, M_{1}\right)$. Let $B^{\prime}$ be $B$ pushed slightly off $M_{1}$ into the interior of $\Sigma_{1}$. Let $\widetilde{\Omega}$ be the result of attaching a (standard) handle of index $k$ to $\Omega$ along $B^{\prime}$. Then $\Omega^{\prime}$ and $\widetilde{\Omega}$ are diffeomorphic.

Proof When we forget about $C$ and $B_{0}$, the pair $\left(H_{\text {right }}, B\right)$ is a standard $(n+1)-$ dimensional handle of index $k$.

For instance, the effect of a right half-handle attachment on $\Omega$ is the same as the effect of a standard handle attachment of the same index.

The situation is completely different in the case of left half-handle attachments.

Lemma 2-19 If $\Omega^{\prime}$ is the result of a left half-handle attachment, then $\Omega^{\prime}$ is diffeomorphic to $\Omega$.

Proof By definition the pair $\left(H_{l e f t}, B\right)$ is diffeomorphic to the pair $\left(D^{n} \times[0,1], D^{n} \times\right.$ $\{0\})$. Attaching $H_{\text {left }}$ along $B$ to $\Omega$ does not change the diffeomorphism type of $\Omega$.

The effect on $Y$ of right and left half-handle attachments are almost the same, the only difference is the index shift by 1 . 
Lemma 2-20 If $\left(\Omega^{\prime}, Y^{\prime} ; \Sigma_{0}, M_{0}, \Sigma_{1}^{\prime}, M_{1}^{\prime}\right)$ is the result of a left (respectively, right) half-handle attachment to $\left(\Omega, Y ; \Sigma_{0}, M_{0}, \Sigma_{1}, M_{1}\right)$ along $\left(B, B_{0}\right) \hookrightarrow\left(\Sigma_{1}, M_{1}\right)$, then $Y^{\prime}$ is the result of a classical handle attachment of index $k-1$ (respectively $k$ ) along $B_{0}$.

Proof This follows immediately from Definition 2-14.

The effects of half handle attachment on $\Sigma$ are also easily described. The next lemma is a direct consequence of the definitions; its proof is omitted. We refer to Figures 5 and 6 .

Lemma 2-21 (a) If $\left(\Omega^{\prime}, Y^{\prime} ; \Sigma_{0}, M_{0}, \Sigma_{1}^{\prime}, M_{1}^{\prime}\right)$ is the result of index $k$ right halfhandle attachment to $\left(\Omega, Y ; \Sigma_{0}, M_{0}, \Sigma_{1}, M_{1}\right)$ along $B_{0} \hookrightarrow M_{1}$, then $\Sigma_{1}^{\prime}$ is diffeomorphic to $\Sigma_{1} \cup_{B_{0}} N$, where $N$ is an $n$-dimensional disk $D^{k} \times D^{n-k}$ and $B_{0}=S^{k-1} \times D^{n-k}$.

(b) Suppose $\left(\Omega^{\prime}, Y^{\prime} ; \Sigma_{0}, M_{0}, \Sigma_{1}^{\prime}, M_{1}^{\prime}\right)$ is the result of left half-handle attachment to $\left(\Omega, Y ; \Sigma_{0}, M_{0}, \Sigma_{1}, M_{1}\right)$ along $\left(B, B_{0}\right) \hookrightarrow\left(\Sigma_{1}, M_{1}\right)$. Then $\Sigma_{1}^{\prime}$ is diffeomorphic to $\Sigma_{1} \backslash B$.

Example 2-22 Suppose $n=3$, so $\Sigma_{1}$ and $\Sigma_{1}^{\prime}$ are three-dimensional manifolds with boundary. The effects on $\Sigma_{1}$ of left and right half-handle attachments to $\Omega$ are the following (the numbers $0,1,2,3,4$ are the index of a handle, "L" and " $\mathrm{R}$ " stand for "left" and "right"):

(0R) $\quad \Sigma_{1}^{\prime}$ is a disjoint union of $\Sigma_{1}$ and a 3-ball.

(1L) A 3-ball is removed from the interior of $\Sigma_{1}$.

(1R) A 1-handle (that is a thickened arc) is added to $\Sigma_{1}$. The attaching region is formed by thickening two point on $\partial \Sigma_{1}$.

(2L) An arc $\gamma$ is chosen inside $\Sigma_{1}$ such that $\partial \gamma \subset \partial \Sigma_{1}$. The manifold $\Sigma_{1}^{\prime}$ is then $\Sigma_{1}$ with a tubular neighborhood of $\gamma$ removed.

(2R) A 2-handle (a thickened two-disk) is added to $\Sigma_{1}$. The attaching region is formed by thickening a circle belonging to $\partial \Sigma_{1}$.

(3L) A disk $D$ is specified inside $\Sigma_{1}$ such that $\partial D \subset \partial \Sigma_{1}$. Then a tubular neighborhood of $D$ is removed.

(3R) A 3-handle (a ball) is added to $\Sigma_{1}$. Notice that adding a 3-ball destroys one component of the boundary.

(4L) A connected component of $\Sigma_{1}$ that is a ball, is removed from $\Sigma_{1}$. This is the opposite of the $(0 \mathrm{R})$ move. 
Lemma 2-21 and Example 2-22 emphasize that right half-handle attachments and left half-handle attachments are somehow dual operations on $\Sigma$. This can be seen also at the Morse function level: changing a Morse function $F$ to $-F$ changes all right half-handles to left-half handles and conversely; see Section 2.4 and 2.5 below. But the above lemma shows another aspect as well: a right half handle attachment consists of gluing a disk, a left half-handle attachment consists of removing a disk. Indeed, in the case of right attachment, $\left(\Sigma_{1}^{\prime}, M_{1}^{\prime}\right)=\left(\Sigma_{1} \cup D^{k} \times D^{n-k}, \partial \Sigma_{1}^{\prime}\right)$ is associated with an embedding $\Phi: \partial D^{k} \times D^{n-k} \rightarrow M_{1}$. On the other hand, by definition, for an embedding $\Phi^{\prime}:\left(D^{k-1} \times D^{n+1-k}, \partial D^{k-1} \times D^{n+1-k}\right) \rightarrow\left(\Sigma_{1}, M_{1}\right)$, the pair

$$
\left(\Sigma_{1}^{\prime}, M_{1}^{\prime}\right)=\left(\text { closure of }\left(\Sigma_{1} \backslash D^{k-1} \times D^{n+1-k}\right), \partial \Sigma_{1}^{\prime}\right)
$$

is obtained from $\left(\Sigma_{1}, M_{1}\right)$ by a handle detachment of index $k-1$. We formulate this observation as a rephrasing of Lemma 2-21.

Corollary 2-23 The effect on $\left(\Sigma_{1}, M_{1}\right)$ of a right half-handle attachment of index $k$ is a handle attachment of index $k$ to $\left(\Sigma_{1}, M_{1}\right)$. Likewise, the effect on $\left(\Sigma_{1}, M_{1}\right)$ of a left half-handle attachment of index $k$ is a handle detachment of index $k-1$. In particular, $M_{1}^{\prime}$ is obtained from $M_{1}$ as the result of a $k$ surgery in the first case, and $k-1$ surgery in the second.

The duality can also be seen as follows: we can cancel any handle attachment by a suitably defined handle detachment, and conversely.

The following definition introduces a terminology which is rather self-explanatory. We include it for completeness of the exposition.

Definition 2-24 A cobordism $\left(\Omega^{\prime}, Y^{\prime}\right)$ between $(\Sigma, M)$ and $\left(\Sigma^{\prime}, M^{\prime}\right)$ is a right (respectively left) half-handle attachment of index $k$ if $\left(\Omega^{\prime}, Y^{\prime}, \Sigma^{\prime}, M^{\prime}\right)$ is a result of right (respectively left) half-handle attachments of index $k$ (in the sense of Definition 2-14) to $(\Sigma \times[0,1], M \times[0,1], \Sigma \times\{0\}, M \times\{0\}, \Sigma \times\{1\}, M \times\{1\})$.

We conclude this section by studying homological properties of handle attachment. These properties will be used in [3]. The proofs are standard and are left to the reader.

Let $\left(H_{+}, C, B, N\right)$ be a half-handle of index $k$.

Lemma 2-25 If $\left(H_{\text {right }}, C, B, N\right)$ is a right half-handle, then the pair $\left(C, B_{0}\right)$ is a strong deformation retract of $\left(H_{+}^{r}, B\right)$, while $\left(D^{k}, \partial D^{k}\right)$ is a strong deformation retract of $\left(C, B_{0}\right)$. In particular, $H_{j}\left(H_{+}^{r}, B\right) \cong H_{j}\left(C, B_{0}\right)=\mathbb{Z}$ for $j=k$, and it is zero otherwise. 
The situation is completely different for left half-handles.

Lemma 2-26 If $\left(H_{\text {left }}, C, B, N\right)$ is a left half-handle, then the pair $\left(H_{\text {left }}, B\right)$ retracts onto the trivial pair (point, point). In particular, all the relative homologies $H_{*}\left(H_{l e f t}, B\right)$ vanish. On the other hand, $\left(D_{0}^{k-1}, S_{0}^{k-2}\right)$ is a strong deformation retract of $\left(C, B_{0}\right)$, hence $H_{j}\left(C, B_{0}\right)=\mathbb{Z}$ for $j=k-1$, and it is zero otherwise. Therefore, the inclusion $\left(C, B_{0}\right) \hookrightarrow\left(H_{\text {left }}, B\right)$ induces a surjection on homologies.

\subsection{Boundary critical points and half-handles}

Consider a Morse function $F$ on a cobordism $(\Omega, Y)$ and assume that it has a single boundary critical point $z$ of index $k$ with critical value $c$ and no interior critical points.

Theorem 2-27 If $z$ is boundary stable (unstable), then the cobordism is a left (right) half-handle attachment of index $k$ respectively.

Proof We can assume that $c=F(z)=0$. Let us chose a neighborhood $U$ of $z$ in $\Omega$. Shrinking $U$ if necessary, we can assume that there are Morse coordinates $x_{1}, \ldots, x_{n+1}$ on $U$ (see Lemma 2-6) and in these coordinates $U$ is a half-ball of radius $2 \rho$ for some positive number $\rho$ :

$$
U=\left\{x_{1}^{2}+\cdots+x_{n+1}^{2} \leqslant 4 \rho^{2}\right\} \cap\left\{x_{1} \geqslant 0\right\} .
$$

The intersection $Y \cap U$ defined by $\left\{x_{1}=0\right\}$, and

$$
F\left(x_{1}, \ldots, x_{n+1}\right)=-a^{2}+b^{2},
$$

where if $z$ is boundary stable we set

$$
a^{2}=x_{1}^{2}+x_{2}^{2}+\cdots+x_{k}^{2}, \quad b^{2}=x_{k+1}^{2}+\cdots+x_{n+1}^{2} \quad(k \geqslant 1),
$$

and if $z$ is boundary unstable

$$
a^{2}=x_{2}^{2}+\cdots+x_{k+1}^{2}, \quad b^{2}=x_{1}^{2}+x_{k+2}^{2}+\cdots+x_{n+1}^{2} \quad(k \geqslant 0) .
$$

We also assume that $x_{1}, \ldots, x_{n+1}$ is an orthonormal Euclidean coordinate system.

Next, we consider $\varepsilon>0$ such that $\varepsilon \ll \rho$, and we define a space $\tilde{H}$ bounded by the following conditions (see Figure 8):

$$
\tilde{H}:=\left\{-a^{2}+b^{2} \in\left[-\varepsilon^{2}, \varepsilon^{2}\right], a^{2} b^{2} \leqslant \rho^{4}-\varepsilon^{4}, x_{1} \geqslant 0\right\} .
$$


Observe that

$$
\tilde{H} \subset U
$$

Let us now define the following parts of the boundary of $\widetilde{H}$ :

$$
\begin{aligned}
\widetilde{B} & =\partial \tilde{H} \cap\left\{-a^{2}+b^{2}=-\varepsilon^{2}\right\} \subset F^{-1}\left(-\varepsilon^{2}\right), \\
\widetilde{P} & =\partial \tilde{H} \cap\left\{-a^{2}+b^{2}=\varepsilon^{2}\right\} \subset F^{-1}\left(\varepsilon^{2}\right), \\
\widetilde{K} & =\partial \tilde{H} \cap\left\{a^{2} b^{2}=\rho^{4}-\varepsilon^{4}\right\}, \\
\widetilde{C} & =\partial \tilde{H} \cap\left\{x_{1}=0\right\} \subset Y .
\end{aligned}
$$

We have $\widetilde{B} \cup \widetilde{P} \cup \widetilde{K} \cup \widetilde{C}=\partial \widetilde{H}$ (in Figure 8 we do not see $\widetilde{C}$, because this would require one more dimension). If $z$ is boundary unstable and $k=0$ in (2-29) then the term $a^{2}$ is missing and $\widetilde{B}=\varnothing$. Otherwise $\widetilde{B} \neq \varnothing$.

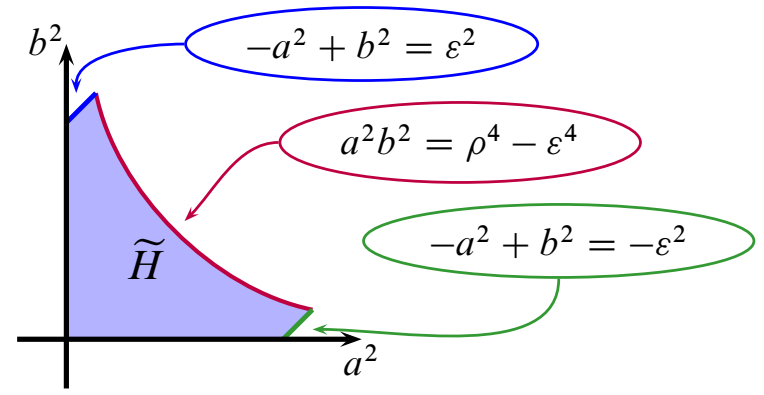

Figure 8: A schematic presentation of $\widetilde{H}, \widetilde{B}, \widetilde{P}, \widetilde{K}$ from the proof of Theorem 2-27. To each point $\left(a^{2}, b^{2}\right)$ in $\widetilde{H}$ on the picture correspond all those points $\left(x_{1}, \ldots, x_{n+1}\right)$ for which (2-28) or (2-29) holds and $x_{1} \geqslant 0$.

Lemma 2-31 The flow of $\nabla F$ is tangent to $\tilde{K}$.

Proof Assume the critical point is boundary stable. The differential equation

$$
\frac{d \boldsymbol{x}}{d t}=\nabla F=\left(-2 x_{1}, \ldots,-2 x_{k}, 2 x_{k+1}, \ldots, 2 x_{n+1}\right)
$$

has a solution

$$
\left(x_{1}, \ldots, x_{n+1}\right) \rightarrow\left(e^{-2 t} x_{1}, \ldots, e^{-2 t} x_{k}, e^{2 t} x_{k+1}, \ldots, e^{2 t} x_{n+1}\right) .
$$

It follows that $a^{2} \rightarrow e^{-4 t} a^{2}$ and $b^{2} \rightarrow e^{4 t} b^{2}$, and the hypersurface $a^{2} b^{2}=$ constant is preserved by the flow of $\nabla F$. 
Lemma 2-32 The inclusion of pairs of spaces

$$
\left(F^{-1}\left(-\varepsilon^{2}\right) \cup_{\widetilde{B}} \tilde{H}, Y \cap\left(F^{-1}\left(-\varepsilon^{2}\right) \cup_{\widetilde{B}} \tilde{H}\right)\right) \subset(\Omega, Y)
$$

admits a strong deformation retract.

Proof By Lemma 2-10 we can assume that

$$
(\Omega, Y)=\left(F^{-1}\left(\left[-\varepsilon^{2}, \varepsilon^{2}\right]\right), Y \cap F^{-1}\left(\left[-\varepsilon^{2}, \varepsilon^{2}\right]\right) .\right.
$$

First we assume that $\widetilde{B}$ is not empty, and it is given by the Equation (2-30) in $U$. Set $\Sigma_{-}=$closure of $\left(F^{-1}\left(-\varepsilon^{2}\right) \backslash \widetilde{B}\right)$ and let $T_{-}$be the part of the boundary of $\Sigma_{-}$ given by

$$
T_{-}=\text {closure of }\left(\partial \Sigma_{-} \backslash \partial F^{-1}\left(-\varepsilon^{2}\right)\right) .
$$

We have $T_{-} \subset \widetilde{B}$; see Figure 9. Let us choose a collar of $T_{-}$in $\Sigma_{-}$, that is a subspace $U_{-} \subset \Sigma_{-}$diffeomorphic to $T_{-} \times[0,1], T_{-}$identified with $T_{-} \times\{0\}$ and $\partial T_{-} \times[0,1] \subset \partial \Sigma_{-} \cap \partial F^{-1}\left(-\varepsilon^{2}\right)$. Let $T_{-}^{\prime}$ be the space identified with $T_{-} \times\{1\}$ by this diffeomorphism.

Similarly, let $\Sigma_{+}=$closure of $\left(F^{-1}\left(\varepsilon^{2}\right) \backslash \widetilde{P}\right)$, and $T_{+}=$closure of $\left(\partial \Sigma_{+} \backslash \partial F^{-1}\left(\varepsilon^{2}\right)\right)$. We also define $\Omega_{0}$ as the closure of $\Omega \backslash \tilde{H}$. Clearly $F$ has no critical points in $\Omega_{0}$ and $\nabla F$ is everywhere tangent to $\partial \Omega_{0} \backslash\left(\Sigma_{-} \cup \Sigma_{+}\right)=\left(Y \cap \Omega_{0}\right) \cup \widetilde{K}$ by Lemma 2-31. In particular, by Lemma 2-10, the flow of $\nabla F$ on $\Omega_{0}$ yields a diffeomorphism between $\Sigma_{-}$and $\Sigma_{+}$, mapping $T_{-}$to $T_{+}$. We define $V \subset \Omega$ as the closure of the set of points $v$ such that a trajectory going through $v$ hits $U_{-}$. Lemma 2-10 implies that there is a diffeomorphism $V \cong T_{-} \times[0,1] \times\left[-\varepsilon^{2}, \varepsilon^{2}\right]$ such that for $(x, t, s) \in V$ we have $F(x, t, s)=s$. Finally, we also define $V^{*}:=\left\{(x, t, s) \in V: s \leqslant \varepsilon^{2}(1-2 t)\right\}$.
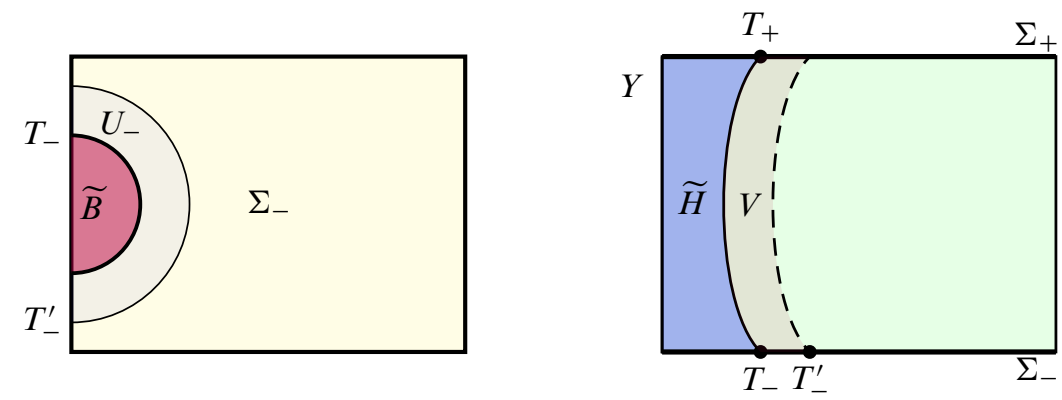

Figure 9: Notation used in Lemma 2-32. Note that the left picture is drawn on $\Sigma_{-}$, while the right one is on $\Omega$.

We define the contraction in two steps: vertical and horizontal. The vertical contraction is defined as follows. For $v \in \widetilde{H} \cup V^{*}$ we define $\Pi_{V}(v)=v$. For a point $v \in \Omega_{0} \backslash V$ 
we take for $\Pi_{V}(v)$ the unique point $s \in \Sigma_{-}$such that a trajectory of $\nabla F$ goes from $s$ to $v$. Finally if $v=(x, t, s) \in V \backslash V^{*}$ we define $\Pi_{V}(v)=\left(x, t, \varepsilon^{2}(1-2 t)\right)$.

By construction, the image of $\Pi_{V}$ is $\tilde{H} \cup V^{*} \cup F^{-1}\left(-\varepsilon^{2}\right)$. Next, we define $\Pi_{H}$. Note that $\Pi_{H}$ will be defined only on the image of $\Pi_{V}$.

$\Pi_{H}=\mathrm{id}$ on $\tilde{H} \cup F^{-1}\left(-\varepsilon^{2}\right)$, and maps $(x, t, s) \in V^{*}$ to $\left(x, t-\left(\varepsilon^{2}+s\right) /\left(2 \varepsilon^{2}\right),-\varepsilon^{2}\right)$ if $s \leqslant \varepsilon^{2}(2 t-1)$, and to $\left(x, 0, s-2 \varepsilon^{2} t\right)$ otherwise. Note that the expressions agree for any $(x, t, s)$ with $s=\varepsilon^{2}(2 t-1)$ and these points are sent to $\left(x, 0,-\varepsilon^{2}\right)$. Both $\Pi_{H}$ and $\Pi_{V}$ are continuous retractions, by smoothing corners we can modify them into smooth retractions; also they can be extended in a natural way to strong deformation retracts. By construction, the retracts preserve $Y$ too. See also Figure 10.

If $\widetilde{B}$ is empty, then $\widetilde{H}$ is necessarily a unstable (right) half-handle of index 0 , $F^{-1}\left(\left[-\varepsilon^{2}, \varepsilon^{2}\right]\right)$ is a disconnected sum of $\tilde{H}$ and the manifold $F^{-1}\left(-\varepsilon^{2}\right) \times\left[-\varepsilon^{2}, \varepsilon^{2}\right]$.
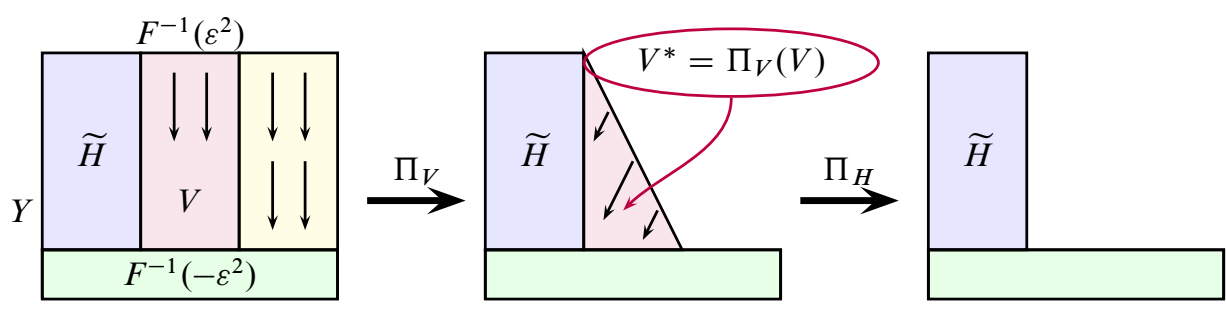

Figure 10: Contractions $\Pi_{H}$ and $\Pi_{V}$ from the proof of Lemma 2-32. The set $V$ is now drawn as a rectangle.

Continuation of the proof of Theorem 2-27 We want to show that $\widetilde{H}$ is a halfhandle.

By Section 2.2 we have the following description in local coordinates of the left half-handle (2-33) and right half-handle (2-34) with cutting coordinate $x_{1}$ :

$$
\begin{aligned}
H_{\text {left }} & =\left\{x_{1}^{2}+\cdots+x_{k}^{2} \leqslant 1\right\} \cap\left\{x_{k+1}^{2}+\cdots+x_{n+1}^{2} \leqslant 1\right\} \cap\left\{x_{1} \geqslant 0\right\}, \\
H_{\text {right }} & =\left\{x_{2}^{2}+\ldots+x_{k+1}^{2} \leqslant 1\right\} \cap\left\{x_{1}^{2}+x_{k+2}^{2}+\ldots+x_{n+1}^{2} \leqslant 1\right\} \cap\left\{x_{1} \geqslant 0\right\} .
\end{aligned}
$$

We consider the subsets $R$ and $S$ of $\mathbb{R}^{2}$ given by

$$
\begin{aligned}
& R=\left\{(u, v) \in \mathbb{R}^{2}: u \geqslant 0, v \geqslant 0, u v \leqslant \rho^{4}-\varepsilon^{4},-u+v \in\left[-\varepsilon^{2}, \varepsilon^{2}\right]\right\}, \\
& S=\left\{(u, v) \in \mathbb{R}^{2}: u \in[0, \varepsilon], v \in[0, \varepsilon]\right\} .
\end{aligned}
$$

(Note that $R$ can be seen in Figure 8 if we replace $a^{2}$ by $u$ and $b^{2}$ by $v$.) These subsets are clearly diffeomorphic. We choose a diffeomorphism $\psi$ that maps the edge 
of $R$ given by $\left\{-u+v=-\varepsilon^{2}\right\}$ to the edge $\{u=\varepsilon\}$ of $S$ and the images of coordinate axes are the corresponding coordinate axes.

We use $\psi$ to construct a diffeomorphism $\Psi$ between $\tilde{H}$ and $H_{\text {right }}$ (respectively $H_{\text {left }}$ ) as follows. First let us write $\psi(u, v)=\left(\psi_{1}(u, v), \psi_{2}(u, v)\right)$. As $\psi$ maps axes to axes, we have $\psi_{1}(0, v)=0$ and $\psi_{2}(u, 0)=0$. Furthermore $\psi_{1}, \psi_{2} \geqslant 0$. By Hadamard's lemma there exist smooth functions $\xi$ and $\eta$ such that

$$
\psi(u, v)=\left(u \xi(u, v)^{2}, v \eta(u, v)^{2}\right) .
$$

We define now

$$
\Psi\left(x_{1}, \ldots, x_{n+1}\right)=\left(\xi(a, b) x_{1}, \ldots, \xi(a, b) x_{k}, \eta(a, b) x_{k+1}, \ldots, \eta(a, b) x_{n+1}\right)
$$

if $z$ is boundary stable, and

$\Psi\left(x_{1}, \ldots, x_{n+1}\right)$

$$
=\left(\eta(a, b) x_{1}, \xi(a, b) x_{2}, \ldots, \xi(a, b) x_{k}, \eta(a, b) x_{k+1}, \ldots, \eta(a, b) x_{n+1}\right)
$$

if $z$ is boundary unstable. Here $a$ and $b$ are given by (2-28) or (2-29). By construction, $\Psi$ maps $(\widetilde{H}, \widetilde{B}, \widetilde{C})$ diffeomorphically to the triple $(H, B, C)$, where

$$
\begin{aligned}
H & =\left\{a^{2} \in\left[0, \varepsilon^{2}\right], b^{2} \in\left[0, \varepsilon^{2}\right], x_{1} \geqslant 0\right\}, \\
B & =\left\{a^{2}=-\varepsilon^{2}, b^{2} \in\left[0, \varepsilon^{2}\right], x_{1} \geqslant 0\right\}, \\
C & =\left\{a^{2} \in\left[0, \varepsilon^{2}\right], b^{2} \in\left[0, \varepsilon^{2}\right], x_{1}=0\right\} .
\end{aligned}
$$

After substituting for $a$ and $b$ the values from (2-28) or (2-29) (depending on whether $z$ is boundary stable or unstable), we recover the model (2-34) of a right half-handle if $z$ is boundary unstable; or the model (2-33) of a left half-handle (both of index $k$ ).

The fact that each half-handle can be presented in a left or right model will be now used to show the following converse to Theorem 2-27. The result for nonboundary case can be found in [12, Theorem 3.12].

Proposition 2-35 Let $(\Omega, Y)=\left(\Sigma_{0} \times[0,1], M_{0} \times[0,1]\right)$ be a product cobordism between $\left(\Sigma_{0}, M_{0}\right)$ and $\left(\Sigma_{1}, M_{1}\right) \cong\left(\Sigma_{0}, M_{0}\right)$. Let us be given a half-handle $(H, C, B)$ of index $k$ and an embedding of $B_{0}=C \cap B$ into $M_{1}$ (respectively an embedding of $\left(B, B_{0}\right)$ into $\left.\left(\Sigma_{1}, M_{1}\right)\right)$, and let $\left(\Omega^{\prime}, Y^{\prime}\right)$ be the result of a right half-handle attachment along $B_{0}$ (respectively, a left half-handle attachment along $\left(B, B_{0}\right)$ ) of index $k$. Then, there exists a Morse function $F:\left(\Omega^{\prime}, Y^{\prime}\right) \rightarrow \mathbb{R}$, which has a single boundary unstable critical point (respectively, a single boundary stable critical point) of index $k$ on $H$ and no other critical points. In particular, $F$ is a Morse function on a cobordism $\left(\Omega^{\prime}, Y^{\prime}\right)$. 
Proof We shall prove the result for right half-handle attachment, the other case is completely analogous. The proof consists mostly on reading "back to front" the proof of Theorem 2-27; we shall use notation from this theorem, with $\varepsilon=1$ and $\rho=2$.

In the case of a right half-handle $B_{0}$ is embedded into $M_{1}$ and we extend this embedding to an embedding of $B$ into $\Sigma_{1}$ (see Definition 2-14 and the remark just after it).

The manifold $\Omega^{\prime}$ is constructed in two steps. First, we glue a handle $\tilde{H}$ to $\Omega=$ $\Sigma_{0} \times[0,1]$ along $B$ obtaining a manifold $\Omega^{\prime \prime}$. The result is as in Figure 10 (the figure on the right). After this gluing, a vertical component of $\partial \tilde{H}$ appears (in notation of (2-30) this vertical component is $\tilde{K})$.

We glue now $\Sigma_{-} \times[-1,1]$ to $\Omega^{\prime \prime}$ so as to obtain $\Omega^{\prime}$ as in Figure 10 on the left and in the way that $\Omega^{\prime \prime}$ is diffeomorphic to $\Omega^{\prime}$. The way we do that is the following. The boundary of $\Sigma_{-} \times[-1,1]$ decomposes into three parts. The first part is $\Sigma_{-} \times\{-1\}$; we glue it to $\Sigma_{0} \times\{1\}$ (notice that $\Sigma_{-}$is $\Sigma_{0}$ with $B$ removed). The second part is $\partial \Sigma_{-} \times[-1,1]$. This part is identified with $\widetilde{K}$, in fact, the flow of $\nabla F$ studied in Lemma 2-31 induces a diffeomorphism of $\tilde{K}$ with $\partial \Sigma_{-} \times[-1,1]$. We glue together $\widetilde{K}$ and $\partial \Sigma_{-} \times[-1,1]$ using this identification. The third part of the boundary, that is, $\Sigma_{-} \times\{1\}$, is not glued. It follows from an argument as in Lemma 2-32 that $\Omega^{\prime}$ is diffeomorphic to $\Omega^{\prime \prime}$.

The manifold $\Omega^{\prime}$ consists of three components: $\Sigma_{0} \times[0,1], H$ and $\Sigma_{-} \times[-1,1]$. We define a function $F$ on each component separately, namely.

$$
F(x)= \begin{cases}t & \text { if } x=(v, t) \in \Sigma_{0} \times\{t\} \subset \Sigma_{0} \times[0,1]=\Omega, \\ 2+t & \text { if } x=(v, t) \in \Sigma_{-} \times\{t\} \subset \Sigma_{-} \times[-1,1], \\ 2-\sum_{j=1}^{k} x_{j}^{2}+\sum_{j=k+1}^{n+1} x_{j}^{2} & \text { if } x=\left(x_{1}, \ldots, x_{n+1}\right) \in H .\end{cases}
$$

As defined, $F$ is smooth on each of the three components. It is also globally continuous. In fact, the identification of $\widetilde{K}$ with $\Sigma_{-} \times[-1,1]$ can be done so that $F$ is continuous on $\tilde{K}$. On the part $\Sigma_{0} \times\{1\} \subset \Omega^{\prime}$, all the three components give the same value, that is 1 .

Given the construction of $F$, it remains to perturb $F$ (that is, to approximate it uniformly near $\left.\tilde{K} \cup \Sigma_{0} \times\{1\}\right)$ to a smooth function and in the way that $F$ does not get any new critical points. For a general piecewise smooth function this is impossible, we can consider the real valued function $x \mapsto|x|$ : any smooth approximation must have a critical point near $x=0$. The reason for this is that near the nonsmooth point the topology of level sets of $|x|$ changes. This is essentially the main obstruction. 
In our situation, the topology of the level sets of $F$ does not change near $\tilde{K}$, nor near $\Sigma_{0} \times\{1\}$, that is, near any gluing region. This is enough to show that $F$ can be approximated near its nonsmooth locus by a smooth function without introducing additional critical points. The proof of this fact is standard, but technical. Instead of giving all the details, we sketch a proof of a weaker result, Lemma 2-36. This result takes care of approximating the function $F$ near $\Sigma_{0} \times\{1\}$. Approximation near the whole of $\tilde{K} \cup \Sigma_{0} \times\{1\}$ follows essentially the same pattern and is left to the reader.

Given the approximation result, the proof of Proposition 2-35 is finished.

Lemma 2-36 (Approximating piecewise smooth functions by smooth functions) Suppose that $N$ is a smooth, compact manifold. Let $\pi: N \times[-1,1] \rightarrow[-1,1]$ be the projection onto the second factor. Let $N_{0}=N \times\{0\}, N_{+}=N \times[0,1]$ and $N_{-}=N \times[-1,0]$. Let $f: N \times[-1,1]$ be a continuous function. Let $f_{+}$and $f_{-}$be the restrictions to $N_{+}$and $N_{-}$respectively. Suppose that

(a) $f_{+}$and $f_{-}$are smooth and have no critical points on $N \times[-1,1]$;

(b) $f_{+}^{-1}(0)=f_{-}^{-1}(0)=N_{0}$;

(c) the image of $f_{+}$is contained in $\mathbb{R}_{\geq 0}$ and the image of $f_{-}$is contained in $\mathbb{R}_{\leq 0}$;

(d) the scalar product $\left\langle f_{ \pm}, \nabla \pi\right\rangle$ is positive on $N_{ \pm} \backslash N_{0}$.

Then for any $\theta>0$ there exist $\varepsilon, \delta \in(0, \theta)$ and a smooth function $g: N \times[-1,1] \rightarrow \mathbb{R}$ such that

(i) $g$ agrees with $f_{-}$on $N \times[-1,-\delta]$ and with $f_{+}$on $N \times[\delta, 1]$;

(ii) $g$ takes values in $[-\varepsilon, \varepsilon]$ on $N \times[-\delta, \delta]$;

(iii) $g$ has no critical points on $N \times[-1,1]$.

Sketch of proof By compactness, the continuity of $f_{ \pm}$and assumptions (b), (c) there exists $\delta^{\prime}>0$ such that $f_{+}\left(N \times\left[0, \delta^{\prime}\right]\right) \subset[0, \theta / 2]$ and $f_{-}\left(N \times\left[-\delta^{\prime}, 0\right]\right) \subset[-\theta / 2,0]$. We set $\varepsilon=\theta$ and $\delta=\min \left(\delta^{\prime}, \theta / 2\right)$.

Choose a partition of unity subordinate to the covering $[-1,1]=[-1,-\delta / 2) \cup(-\delta, \delta) \cup$ $(\delta / 2,1]$. The three functions corresponding to this partition are denoted by $\phi_{-}, \phi_{0}$ and $\phi_{+}$respectively.

Define $\Phi_{\bullet}: N \times[-1,1] \rightarrow[0,1]$ as compositions $\phi_{\bullet} \circ \pi$, where $\bullet$ is any of “+”, "-" and " 0 ". Consider the vector field

$$
v=\Phi_{+} \nabla f_{+}+\Phi_{0} \nabla \pi+\Phi_{-} \nabla f_{-} .
$$


By point (a) of the assumptions $v$ is a smooth vector field. Assumption (d) implies that $\langle\nabla \pi, v\rangle>0$ everywhere on $N \times[-1,1]$, that is, $v$ is a gradient-like vector field for $\pi$. In particular, $v$ does not vanish on $N \times[-1,1]$.

Let $h$ be a positive $C^{\infty}$ function. Set $v_{h}=h v$. Then $v_{h}$ is also a gradient-like vector field for $\pi$. The trajectories of $v$ coincide with those of $v_{h}$ : multiplication by $h$ changes only the speed of going along a trajectory.

We integrate the vector field $v_{h}$ to a function $g_{h}: N \times[-1,1] \rightarrow \mathbb{R}$. This means we first set $g_{h} \equiv f_{-}$on $N \times\{-1\}$. Next, suppose $x \in N \times(-1,1]$. Let $\gamma: U \rightarrow N \times[-1,1]$ (here $U$ is a closed interval) be a trajectory of $v_{h}$ such that $\gamma(0)=x$. Since $v_{h}$ is gradient-like for $\pi, \gamma$ must have come from $N \times\{-1\}$ in the past, more precisely, there exist $t_{x}<0$ and $y \in N \times\{-1\}$ such that $\gamma\left(t_{x}\right)=y$. We set

$$
g_{h}(x)=g_{h}(y)-t_{x} .
$$

Since $v_{h}$ is smooth, by the implicit function theorem $g_{h}$ is a smooth function.

Choosing the normalizing function $h$ appropriately we can guarantee that $g:=g_{h}$ satisfies (i). Namely, we set $h=\left\|\nabla f_{-}\right\|^{-2}$ so that the directional derivative $\left\langle v_{h}, \nabla f_{-}\right\rangle \equiv 1$ on $N \times[-1,-\delta]$. This implies that $g=f_{-}$on $N \times[-1,-\delta]$. The choice of $h$ on $N \times[-\delta, \delta]$ is such that the time the trajectory goes from a point $x_{-} \in N \times\{-\delta\}$ to some $x_{+} \in N \times\{\delta\}$ is equal to $f_{+}\left(x_{+}\right)-f_{-}\left(x_{-}\right)$. The latter expression is positive by assumption (b). This implies that $g=f_{+}$on $N \times\{\delta\}$ and condition (ii) is satisfied automatically. Finally we set $h=\left\|\nabla f_{+}\right\|^{-2}$ on $N \times[\delta, 1]$. The verification of condition (i) is straightforward.

As $g_{h}$ is strictly increasing on trajectories of $v_{h}$, it cannot have any critical points.

\subsection{Left and right product cobordisms and traces of handle attachments}

In this subsection we create a dictionary between surgery theoretical notions (traces of handle attachments and detachments) and Morse theoretical (additions of half-handles). The main result of this subsection, Proposition 2-38, is a direct consequence of the results proved earlier in the article.

To begin with, let $(\Omega, Y)$ be a cobordism between $\left(\Sigma_{0}, M_{0}\right)$ and $\left(\Sigma_{1}, M_{1}\right)$.

Definition 2-37 We shall say that $\Omega$ is a left product cobordism if $\Omega \cong \Sigma_{0} \times[0,1]$. Similarly, if $\Omega \cong \Sigma_{1} \times[0,1]$, then we shall say that $\Omega$ is a right product cobordism. 
Proposition 2-38 (a) If $(\Omega, Y)$ is a cobordism between $\left(\Sigma_{0}, M_{0}\right)$ and $\left(\Sigma_{1}, M_{1}\right)$ consisting only of left half-handle attachments, then it is a left-product cobordism. Likewise, if it consists only of right half-handle attachments, then it is a right product cobordism.

(b) Let $F: \Omega \rightarrow[0,1]$ be a Morse function in the sense of Definition 1-4. Assume that $F$ has no critical points in the interior of $\Omega$. If all critical points on the boundary are boundary stable, then $F$ is a left-product cobordism. If all critical points are boundary unstable, then $F$ is a right product cobordism.

Proof Statements (a) and (b) are equivalent via Theorem 2-27 and Proposition 2-35. The stable-unstable (right-left) statements are also equivalent by replacing the Morse function $F$ by $-F$. The stable case follows from Lemma 2-19.

The next results of this subsection will be not used in this paper, but we add them because they bridge surgery techniques and applications, eg with [14] or [3].

In order to clarify what we wish, let us recall that by Theorem 2-27 if a Morse function $F$ defined on a cobordism $(\Omega, Y)$ has only one critical point of boundary type then $(\Omega, Y)$ is a half-handle attachment. Proposition 2-35 is the converse of this; the (total) space of a half-handle attachment can be thought as a cobordism with a Morse function on it with only one critical point.

We wish to establish the analogues of these statements "at the level of $\Sigma$ ". In Section 2.3 we proved that the output of a right/left half-handle attachment induces a handle attachment/detachment at the level of $\Sigma$. The next lemma is the converse of this statement. (In fact, the output cobordism provided by it can be identified with the cobordism constructed in Proposition 2-35.)

Lemma 2-39 Assume that $\left(\Sigma_{1}, M_{1}\right)$ is the result of a handle attachment (respectively detachment) to $\left(\Sigma_{0}, M_{0}\right)$. Then, there exists a cobordism $\left(\Omega, Y ; \Sigma_{0}, M_{0}, \Sigma_{1}, M_{1}\right)$ such that $\Omega \cong \Sigma_{1} \times[0,1]$ (respectively $\Omega \cong \Sigma_{0} \times[0,1]$ ).

Proof Assume that $\left(\Sigma_{1}, M_{1}\right)$ arises from a handle attachment to $\left(\Sigma_{0}, M_{0}\right)$, ie $\Sigma_{1}=$ $\Sigma_{0} \cup D^{k} \times D^{n-k}$. Let us define $\Omega=\Sigma_{1} \times[0,1]$. The boundary $\partial \Omega$ can be split as

$$
\begin{aligned}
\partial \Omega & =\left(\Sigma_{0} \cup D^{k} \times D^{n-k}\right) \times\{0\} \cup\left(M_{1} \times[0,1]\right) \cup\left(\Sigma_{1} \times\{1\}\right) \\
& =\Sigma_{0} \times\{0\} \cup Y \cup \Sigma_{1} \times\{1\},
\end{aligned}
$$

where $Y=D^{k} \times D^{n-k} \cup\left(M_{1} \times[0,1]\right)$. Its $D^{k} \times D^{n-k}$ part can be "pushed inside" $\Omega$ transforming (diffeomorphically) $\Omega$ into a cobordism; see Figure 11 . 
An analogous construction can be used in the case of a handle detachment. If $\left(\Sigma_{1}^{\prime}, M_{1}^{\prime}\right)$ is the result of a handle detachment from $\left(\Sigma_{0}, M_{0}\right)$, then the trace of the handle detachment is the cobordism between $\left(\Sigma_{0}, M_{0}\right)$ and $\left(\Sigma_{1}^{\prime}, M_{1}^{\prime}\right)$ such that

$$
\left(\Omega^{\prime}, Y^{\prime}\right)=\left(\Sigma_{0} \times[0,1], M_{0} \times[0,1] \cup D^{k} \times D^{n-k}\right) .
$$
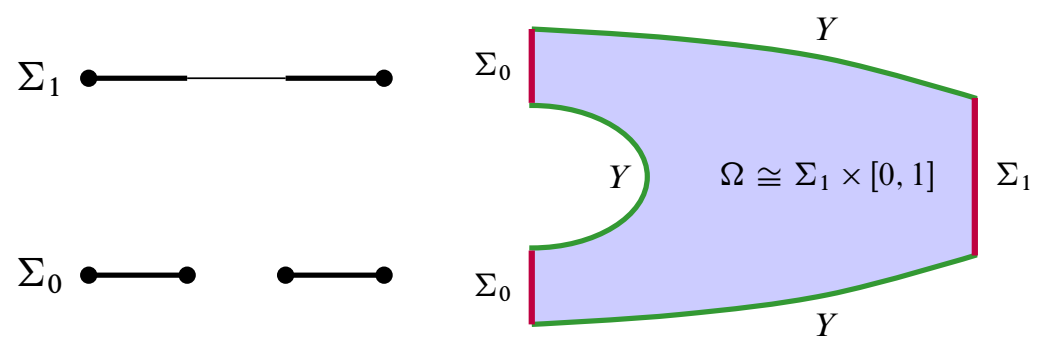

Figure 11: Lemma 2-39. On the left a 1-handle is attached to $\Sigma_{0}$. On the right there is a cobordism between $\Sigma_{0}$ and $\Sigma_{1}$, which is a right product cobordism.

Definition 2-40 The cobordism $\left(\Omega, Y ; \Sigma_{0}, M_{0}, \Sigma_{1}, M_{1}\right)$ determined by Lemma 2-39 is called the trace of a handle attachment of $\left(\Sigma_{0}, M_{0}\right)$ (respectively the trace of a handle detachment).

\section{Splitting interior handles}

We prove here the theorem about moving critical points to the boundary.

Theorem 3-1 Assume that on a cobordism $(\Omega, Y)$ between $\left(\Sigma_{0}, M_{0}\right)$ and $\left(\Sigma_{1}, M_{1}\right)$ we have a Morse function $F$ with a single critical point $z$ of index $k \in\{1, \ldots, n\}$ in the interior of $\Omega$ situated on the level set $\Sigma_{1 / 2}=F^{-1}(F(z))$. If

(3-2) the connected component of $\Sigma_{1 / 2}$ containing $z$ has nonempty intersection with $Y$,

then there exists a function $G: \Omega \rightarrow[0,1]$, such that:

- $G$ agrees with $F$ in a neighborhood of $\Sigma_{0} \cup \Sigma_{1}$.

- $\nabla G$ is everywhere tangent to $Y$.

- $G$ has exactly two critical points $z^{s}$ and $z^{u}$, which are both on the boundary and of index $k$. The point $z^{s}$ is boundary stable and $z^{u}$ is boundary unstable.

- There exists a Riemannian metric such that there is a single trajectory of $\nabla G$ from $z^{s}$ to $z^{u}$ inside $Y$. 
Remark 3-3 A careful reading of the proof shows that we can in fact construct a smooth homotopy $G_{t}$ such that $F=G_{0}, G=G_{1}$ and there exists $t_{0} \in(0,1)$ such that $G_{t}$ has a single interior critical point for $t<t_{0}$, two boundary critical points for $t>t_{0}$ and a degenerate critical point on the boundary for $t=t_{0}$. See Remark 3-15.

The proof of Theorem 3-1 occupies Sections 3.2 to 3.4. We make a detailed discussion of Condition (3-2) in Section 3.5.

\subsection{About the proof}

The argument is based on the following two-dimensional picture. Consider the set $Z=\left\{(x, y) \in \mathbb{R}^{2}: x \geqslant 0\right\}$ and the function $D: Z \rightarrow \mathbb{R}$ given by

$$
D(x, y)=y^{3}-y x^{2}+a y,
$$

where $a \in \mathbb{R}$ is a parameter. Observe that the boundary of $Z$ given by $\{x=0\}$ is invariant under the gradient flow of $D$ (see Figure 12).

Lemma 3-4 For $a>0, D$ has a single Morse critical point in the interior of $Z$. For $a<0, D$ has two Morse critical points on the boundary of $Z$.

Proof Critical points of $D$ are given by $\frac{\partial D}{\partial x}=\frac{\partial D}{\partial y}=0$, that is, $x y=0$ and $3 y^{2}-$ $x^{2}+a=0$. The first equation means that $y=0$ or $x=0$ and then we get solutions $( \pm \sqrt{a}, 0)$ and $(0, \pm \sqrt{-a / 3})$. In the case $a>0$ we consider only first two solutions (and only one of them belongs to $Z$ ), while if $a<0$, only the last two solutions are real and they correspond to boundary critical points. Checking that these critical points are Morse is straightforward and is left to the reader.

For $a=0, D$ acquires a $D_{4}^{-}$singularity at the origin (see eg [1, Section 17.1]).

In the proof of Theorem 3-1, we start by introducing "local/global" coordinates $\left(x, y, u_{1}, \ldots, u_{n-1}\right)$ at $z$, in which $F$ has the form $D(x, y) \pm u_{1}^{2} \pm \cdots \pm u_{n-1}^{2}$, hence it also parametrizes a neighborhood of a path connecting $z$ with a point of $Y$. Then we change the parameter $a$ (which we originally assume to be equal to 1 ) to $-\delta$, where $\delta$ is very small positive number (which corresponds to moving the critical point to the boundary along the chosen path).

\subsection{Proof of Theorem 3-1 under an additional assumption}

We first give the proof assuming the existence of such coordinate system as in 3.1, described explicitly in the next proposition (which is proved in Section 3.4). We use the hypotheses and notation of Theorem 3-1. 

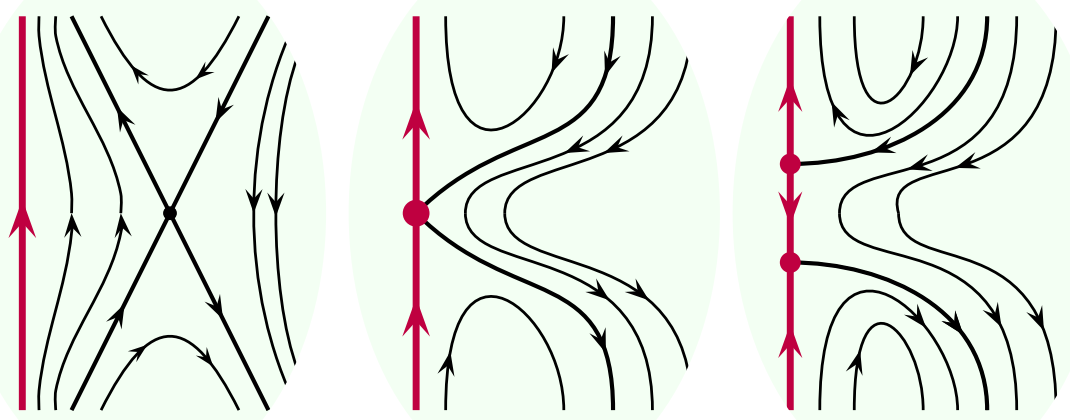

Figure 12: The trajectories of the gradient vector field of $D$ for values of $a>0, a=0$ and $a<0$.

Proposition 3-5 There exists $\eta>0, \eta \ll 1$ and an open "half-disk" $U \subset \Omega$, intersecting $Y$ along a disk, and coordinates $x, y, u_{1}, \ldots, u_{n-1}$ such that in these coordinates $U$ is given by

$$
0 \leqslant x<3+\eta, \quad|y|<\eta, \quad \sum_{j=1}^{n-1} u_{j}^{2}<\eta^{2},
$$

$U \cap Y$ is given by $\{x=0\}$, and in these coordinates $F$ is given by

$$
y^{3}-y x^{2}+y+\frac{1}{2}+\sum_{j=1}^{n-1} \epsilon_{j} u_{j}^{2},
$$

where $\epsilon_{1}, \ldots, \epsilon_{n-1} \in\{ \pm 1\}$ are choices of signs. In particular \#\{j: $\left.\epsilon_{j}=-1\right\}=k-1$, where $k=\operatorname{ind}_{z} F$.

Assuming the proposition, we prove Theorem 3-1. Let us introduce some abbreviations:

$$
\vec{u}=\left(u_{1}, \ldots, u_{n-1}\right), \quad \vec{u}^{2}=\sum_{j=1}^{n-1} \epsilon_{j} u_{j}^{2}, \quad\|\vec{u}\|^{2}=\sum_{j=1}^{n-1} u_{j}^{2} .
$$

We fix a small real number $\varepsilon>0$ such that $\varepsilon \ll \eta$ and two subsets $U_{1} \subset U_{2}$ of $U$ by

$$
\begin{aligned}
& U_{1}=\{|y| \leqslant \varepsilon, x \leqslant 3\} \cup\left\{(x-3)^{2}+y^{2} \leqslant \varepsilon^{2}\right\}, \\
& U_{2}=\{|y| \leqslant 2 \varepsilon, x \leqslant 3\} \cup\left\{(x-3)^{2}+y^{2} \leqslant 4 \varepsilon^{2}\right\} .
\end{aligned}
$$

The difference $U_{21}:=\overline{U_{2} \backslash U_{1}}$ splits into two subsets $S_{1} \cup S_{2}$ (see Figure 13), where

$$
S_{1}=U_{21} \cap\{x \leqslant 3\}, \quad S_{2}=U_{21} \cap\{x \geqslant 3\} .
$$




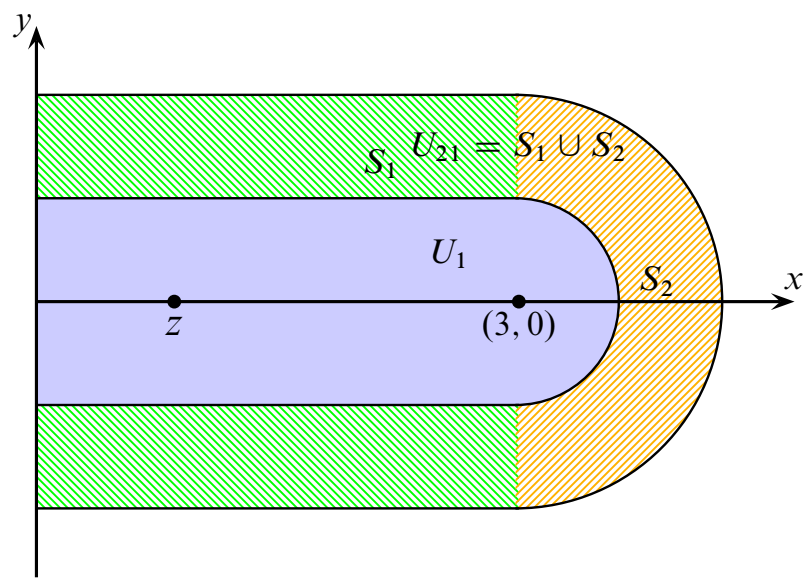

Figure 13: Sets $U_{1}, U_{21}, S_{1}$ and $S_{2}$ in two dimensions (coordinates $x$ and $y$ ).

For a point $v=\left(x, y, u_{1}, \ldots, u_{n-1}\right) \in U$, let us define

$$
\widetilde{s}(v)= \begin{cases}1 & \text { if } v \in U_{1}, \\ 0 & \text { if } v \in \overline{U \backslash U_{2},} \\ 2-\frac{|y|}{\varepsilon} & \text { if } v \in S_{1}, \\ 2-\frac{\sqrt{(x-3)^{2}+y^{2}}}{\varepsilon} & \text { if } v \in S_{2} .\end{cases}
$$

The above formula defines a continuous function $\tilde{s}: U_{2} \rightarrow[0,1]$. It is smooth away of $\partial S_{1} \cup \partial S_{2}$. We can perturb it to a $C^{\infty}$ function $s: U_{2} \rightarrow[0,1]$, with the following properties:

(S1) $s^{-1}(1)=U_{1}, s^{-1}(0)=\left\{|y| \geqslant 2 \varepsilon-\varepsilon^{2}\right\} \cup\left\{(x-3)^{2}+y^{2} \geqslant 4 \varepsilon^{2}-\varepsilon^{3}, x \geq 3\right\}$ (this is a thin region near the boundary of $U_{2}$ ).

(S2) $\frac{\partial s}{\partial u_{j}}=0$ for any $j=1, \ldots, n-1$.

(S3) $\frac{\partial s}{\partial x}=0$, and $\left|\frac{\partial s}{\partial y}\right|<2 / \varepsilon$ at all points of $S_{1}$. Furthermore $y \frac{\partial s}{\partial y}<0$ at all points of $S_{1}$.

(S4) If $v \in S_{2}$ and we choose radial coordinates $x=3+r \cos \theta, y=r \sin \theta$ (where $r \in[\varepsilon, 2 \varepsilon]$ and $\theta \in[-\pi / 2, \pi / 2])$, then $\left|\frac{\partial s}{\partial r}\right|<\frac{2}{\varepsilon}$ and $\left|\frac{\partial s}{\partial \theta}\right|<\varepsilon$

Observe that $\tilde{S}$ satisfies (S1)-(S4) at every point for which it is smooth; the only issue is that on $S_{1} \cap S_{2}, \tilde{s}$ fails to be $C^{2}$.

Now let us choose a smooth decreasing function $\phi:\left[0, \eta^{2}\right] \rightarrow[0,1]$, which is equal to 0 on $\left[\frac{3}{4} \eta^{2}, \eta^{2}\right]$ and $\phi(0)=1$. We define now a new function $b: U_{2} \rightarrow[0,1]$ by the 
formula

$$
b(x, y, \vec{u})=s(x, y, \vec{u}) \cdot \phi\left(\|\vec{u}\|^{2}\right) .
$$

Let us finally define the function $G: \Omega \rightarrow[0,1]$ by

$$
(3-8) \quad G(w)= \begin{cases}F(w) & \text { if } w \notin U_{2} \\ y^{3}-y x^{2}+y-(\delta+1) b(x, y, \vec{u}) y+\frac{1}{2}+\vec{u}^{2} & \text { if } w=(x, y, \vec{u}) \in U_{2},\end{cases}
$$

where $\delta>0$ is a very small number. Later we shall show that it is enough to take $\delta<\varepsilon^{2} / 2$. In the following lemmas we shall prove that $G$ satisfies the conditions of Theorem 3-1.

Lemma 3-9 The function $G$ is smooth.

Proof It is a routine checking and we leave it for the reader.

In the next two lemmas we show that $G$ has no critical points in $U_{21}$.

Lemma 3-10 $G$ has no critical points on $U_{21} \cap\{y=0\}$.

Proof If $\left(x, 0, u_{1}, \ldots, u_{n-1}\right) \in U_{21}$ then $x>3$. Consider the derivative over $y$ of $G$ :

$$
\frac{\partial G}{\partial y}=3 y^{2}-x^{2}+1-(\delta+1) b-(\delta+1) \phi\left(u_{1}^{2}+\cdots+u_{n-1}^{2}\right) \frac{\partial s}{\partial y} y .
$$

Taking $y=0$ we get $-x^{2}+1-(\delta+1) b$. Since $b$ takes values in $[0,1]$ and $x>3$, one gets $\frac{\partial G}{\partial y}<0$.

Lemma 3-12 If $\delta<3 \varepsilon^{2}$, then $G$ has no critical points on $U_{21} \cap\{y \neq 0\}$.

Proof Assume that $\frac{\partial G}{\partial x}=0$ for some $(x, y, \vec{u})$. Then

$$
y\left(-2 x-(\delta+1) \frac{\partial s}{\partial x} \phi\right)=0 .
$$

As $y \neq 0$, the expression in parentheses should be zero. If $0<x \leqslant 3$, then by (S3) we have $\frac{\partial s}{\partial x}=0$. Hence the above equality can not hold. Assume that $x=0$. In the derivative over $y$ (see Equation (3-11)), the expression $-(\delta+1) \phi \frac{\partial s}{\partial y} y$ is nonnegative by (S3). Furthermore $b<1$, hence

$$
\frac{\partial G}{\partial y} \geqslant 3 y^{2}-\delta .
$$


Now if $\delta<3 \varepsilon^{2}$ then there are no critical points with $x=0$. It remains to deal with the case $\left(x, y, u_{1}, \ldots, u_{n-1}\right) \in S_{2}$. Consider the derivative $\frac{\partial G}{\partial y}$. By (S4) and the chain rule we have

$$
\left|y \frac{\partial s}{\partial y}\right|=\left|y \frac{\partial r}{\partial y} \frac{\partial s}{\partial r}+y \frac{\partial \theta}{\partial y} \frac{\partial s}{\partial \theta}\right| \leq\left|\frac{y^{2}}{r} \cdot \frac{\partial s}{\partial r}\right|+\left|\frac{(x-3) y}{r^{2}} \cdot \frac{\partial s}{\partial \theta}\right|<r \frac{2}{\varepsilon}+\varepsilon<5 .
$$

Furthermore $|1-(\delta+1) b| \leqslant 1$, and $\left|3 y^{2}\right|<1$ because $\varepsilon$ is small. As $x \geqslant 3$, we have $\frac{\partial G}{\partial y}<0$ on $S_{2}$.

On $U_{1}$ the function $G$ is given by

$$
G(x, y, \vec{u})=y^{3}-y x^{2}-\delta y+\vec{u}^{2}+\frac{1}{2} .
$$

As in Section 3.1 we study the critical points in $U_{1}$.

Lemma 3-14 $G$ has two critical points on $U_{1}$ at

$$
\begin{aligned}
& z^{s}:=(0, \sqrt{\delta / 3}, 0, \ldots, 0), \\
& z^{u}:=(0,-\sqrt{\delta / 3}, 0, \ldots, 0) .
\end{aligned}
$$

Both critical points are boundary, both of Morse index $k, z^{s}$ is stable, while $z^{u}$ is unstable.

Proof The derivative of $G$ vanishes only at $z^{s}$ and $z^{u}$. Indices are immediately computed from (3-13). The point $z^{s}$ is boundary stable, because for $z^{s}$ the expression $-y x^{2}$ is negative and the boundary is given by $x=0$, hence it is attracting in the normal direction. Similarly we prove for $z^{u}$. See also Figure 12 for the two-dimensional picture.

Remark 3-15 If we define $G_{t}=y^{3}-y x^{2}+y-t(\delta+1) b \cdot y+\frac{1}{2}+\vec{u}^{2}$ for $t \in[0,1]$, then the same argument as in Lemmas 3-10 and 3-12 shows that $G_{t}$ has no critical points in $U_{2} \backslash U_{1}$. As for critical points in $U_{1}$, observe that on $U_{1}$ we have

$$
G_{t}=y^{3}-y x^{2}+(1-t(1+\delta)) y+\frac{1}{2}+\vec{u}^{2} .
$$

Let $t_{0}=1 /(1+\delta)$. If $t>t_{0}$, the function $G_{t}$ has two critical points on the boundary $Y$, while for $t<t_{0}, G_{t}$ has a single critical point in the interior $U_{1} \backslash Y$. If $t=t_{0}$, $G_{t}$ has a single degenerate critical point on $Y$. In this way we construct an "isotopy" between $F$ and $G$. 
Let us now choose a Riemannian metric $g^{\prime}$ on

$$
U_{1}^{\prime}:=U_{1} \cap\{\|\vec{u}\|<\varepsilon\}
$$

by the condition that $\left(x, y, u_{1}, \ldots, u_{n-1}\right)$ be orthonormal coordinates (cf Remark 3-18 below). Clearly, any metric $g$ on $\Omega$ can be changed near $U_{1}$ so as to agree with $g^{\prime}$ on $U_{1}^{\prime}$. In this metric the gradient of $G$ is

$$
\left(-2 x y, 3 y^{2}-x^{2}-\delta, 2 \epsilon_{1} u_{1}, \ldots, 2 \epsilon_{n-1} u_{n-1}\right) .
$$

We want to show that there is a single trajectory starting from $z^{s}$ and terminating at $z^{u}$. Clearly, there is one trajectory from $z^{s}$ to $z^{u}$ which stays in $U_{1}^{\prime}$ (having $y=0$ and $\vec{u}=0$ ). In order to eliminate the others, we need the following lemma.

Lemma 3-17 Let $\gamma$ be a trajectory of $\nabla G$ starting from $z^{s}$. Let $w$ be the point, where $\gamma$ hits $\partial U_{1}^{\prime}$ for the first time. If $\delta$ is sufficiently small, then $G(w)>G\left(z^{u}\right)$.

Proof Assume that $\gamma(t)$ is such trajectory. Assume that among numbers $\epsilon_{i}$, we have $\epsilon_{i}=-1$ for $i \leqslant k-1$ and $\epsilon_{i}=1$ otherwise. As $z^{s}$ is a critical point of the vector field $\nabla G$ with a nondegenerate linear part, we conclude that the limit

$$
\lim _{t \rightarrow-\infty} \frac{\gamma^{\prime}(t)}{\left\|\gamma^{\prime}(t)\right\|}=: v=\left(x_{0}, y_{0}, u_{01}, \ldots, u_{0, n-1}\right)
$$

exists. The vector $v$ is the tangent vector to the curve $\gamma$ at the point $z^{s}$, and it lies in the unstable space. Hence $x_{0}=0$ as $(1,0, \ldots, 0)$ is a stable direction; similarly $u_{01}=\cdots=u_{0, k-1}=0$. Therefore, until $\gamma$ hits the boundary of $U_{1}^{\prime}$ for the first time, we have

$$
x=u_{1}=\cdots=u_{k-1}=0 .
$$

Set also $g(y)=y^{3}-\delta y$. One has the following cases, depending the position of $w$, where $\gamma$ hits $\partial U_{1}^{\prime}$ for the first time: (a) $y=-\varepsilon$, (b) $y=\varepsilon$, or (c) $\|\vec{u}\|^{2}=\eta^{2}$. The case (a) cannot happen since $G$ is increasing along the trajectory, hence $G(w)>$ $G\left(z^{s}\right)$, a fact which contradicts $g(-\varepsilon)<g(\sqrt{\delta / 3})$ valid for $2 \delta<\varepsilon^{2}$. In case (b), $G(w)>G\left(z^{u}\right)$ follows from $g(\varepsilon)>g(-\sqrt{\delta / 3})$. Finally, assume the case (c). Then, as $u_{01}=\cdots=u_{0, k-1}=0$, we obtain $\vec{u}^{2}=\|\vec{u}\|^{2}=\eta^{2}$. Then $G(w)-G\left(z^{s}\right) \geqslant \eta^{2}$, because the contribution to $G$ from $y^{3}-\delta y$ increases along $\gamma$. Hence $G(w)>G\left(z^{u}\right)$ follows again since $\varepsilon \ll \eta$.

Given the above lemma it is clear that if a trajectory $\gamma$ leaves $U_{1}^{\prime}$, then $G$ becomes bigger than $G\left(z^{u}\right)$. As $G$ increases along any trajectory, it is impossible that such trajectory limits in $z^{u}$. The proof of Theorem 3-1, up to Proposition 3-5, is accomplished. 
Remark 3-18 The metric $g^{\prime}$ defined below Remark 3-15 can be chosen so that $(x, y, \vec{u})$ forms an orthogonal, but not necessarily orthonormal coordinate system. Each component of the vector field (3-16) is then multiplied by a positive constant, the statement of Lemma 3-17 still holds with essentially the same proof. However, $g^{\prime}$ cannot be just any metric; we can choose a metric $g^{\prime}$ in a way that there is an arbitrary number of trajectories from $z^{s}$ to $z^{u}$ (topologically changing the metric can produce a pair of mutually canceling intersection points between the unstable manifold of $z^{s}$ and the stable manifold of $z^{u}$ ).

\subsection{An auxiliary construction.}

The following construction is a crucial ingredient in the proof of Proposition 3-5; see the next section. Set

$$
Z=\left\{(x, y) \in \mathbb{R}^{2}: x \geqslant 0\right\},
$$

and define the two functions

$$
A(x, y)=\frac{x^{3}}{3 \sqrt{3}}-\sqrt{3} x y^{2}-\frac{x}{\sqrt{3}}+\frac{2}{3 \sqrt{3}}, \quad B(x, y)=y^{3}-y x^{2}+y .
$$

Observe that

$$
A+i B=\left(\frac{x}{\sqrt{3}}-i y\right)^{3}-\left(\frac{x}{\sqrt{3}}-i y\right)+\frac{2}{3 \sqrt{3}} .
$$

Up to a linear transformation, the map $(x, y) \mapsto A+i B$ is a holomorphic map. Thus it shares several geometric properties of a holomorphic map. For example, it is an open map, and the singular points are precisely the points where the gradient of $B$ vanishes. Let us choose $\delta>0$ smaller than $2 /(3 \sqrt{3})$. Consider two sets

$$
\begin{aligned}
& Z_{1}=\{(x, y) \in Z, x<1, A(x, y) \geq \delta\}, \\
& Z_{2}=\{(x, y) \in Z, x>1, A(x, y) \geq \delta\} .
\end{aligned}
$$

We have the following result.

Lemma 3-21 The map $\psi(x, y)=(A(x, y), B(x, y))$ maps $Z_{1}$ and $Z_{2}$ diffeomorphically onto $E_{1}$ and $E_{2}$ respectively, where

$$
E_{1}=\left\{(a, b) \in \mathbb{R}^{2}: a \in\left[\delta, \frac{2}{3 \sqrt{3}}\right]\right\}, \quad E_{2}=\left\{(a, b) \in \mathbb{R}^{2}: a \geq \delta\right\} .
$$

Proof One readily checks that $\psi: Z_{1} \rightarrow V_{1}$ and $\psi: Z_{2} \rightarrow V_{2}$ are bijections. As the derivative $D \psi$ is nondegenerate on $Z_{1} \cup Z_{2}, \psi$ is a diffeomorphism between the two pairs of sets. 


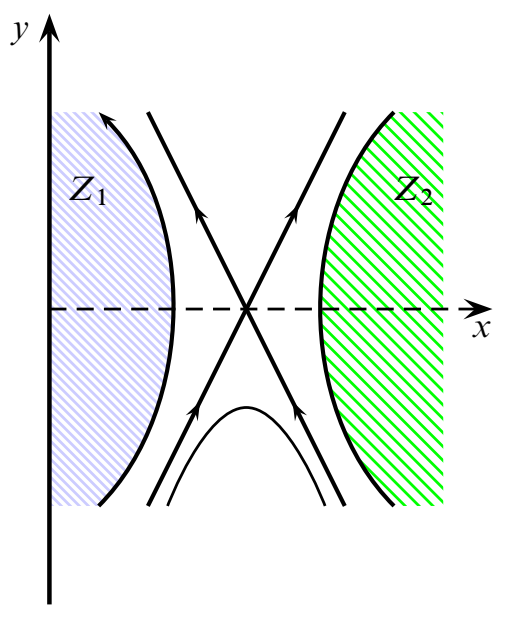

Figure 14: Sets $Z_{1}$ and $Z_{2}$ from Section 3.3. There is also drawn the singular level set $A^{-1}(0)$.

\subsection{Proof of Proposition 3-5}

First, as $z$ is a critical point of index $k \in\{1, \ldots, n\}$, by the Morse Lemma 2-6 we can find a neighborhood $\tilde{V}$ of $z$ and a chart $h_{1}: \tilde{V} \rightarrow \mathbb{R}^{n+1}$, with coordinates $\left(x^{\prime}, y, \vec{u}\right)$ such that

$$
F \circ h_{1}^{-1}\left(x^{\prime}, y, \vec{u}\right)=x^{\prime} y+\vec{u}^{2}+\frac{1}{2}
$$

Remark 3-22 The term $x^{\prime} y$ (corresponding to a hyperbolic quadratic form) is the moment when the assumption that $k \neq 0, n+1$ is used.

Let us define a map $h_{2}(x, y, \vec{u})=\left(x^{\prime}, y, \vec{u}\right)$, where $x^{\prime}=y^{2}+1-x^{2}$. By the inverse function theorem, $h_{2}$ is a local diffeomorphism near $(1,0, \ldots, 0)$. Shrinking $\tilde{V}$ if needed, and considering $h_{3}=h_{2}^{-1} \circ h_{1}$, we obtain $h_{3}(z)=(1,0, \ldots, 0)$ and

$$
F \circ h_{3}^{-1}(x, y, \vec{u})=y^{3}-y x^{2}+y+\vec{u}^{2}+\frac{1}{2}=B(x, y)+\vec{u}^{2}+\frac{1}{2} .
$$

Let us pick now $\xi>0$ such that the cylinder

$$
V=\{|x-1|<\xi,|y|<\xi,\|\vec{u}\|<\xi\} \subset \mathbb{R}^{n+1}
$$

lies entirely in $h_{3}(\tilde{V})$. By shrinking $\tilde{V}$ we may in fact assume that $h_{3}(\tilde{V})=V$. If $0<\delta \ll 2 /(3 \sqrt{3})$ is sufficiently small then $A(x, 0)<\delta$ implies $|x-1|<\xi$. Choose such a $\delta$, and set

$$
V_{1}:=V \cap\{x<1, A(x, y) \geq \delta\}
$$


(compare (3-20)). By Lemma 3-21 the map

$$
\Psi_{1}(x, y, \vec{u})=\left(A(x, y), B(x, y)+\vec{u}^{2}, \vec{u}\right),
$$

is a diffeomorphism (being the composition of $\psi \oplus \operatorname{Id}_{\mathbb{R}^{n+1}}$ and a "triangular" map). Set $C_{1}:=\Psi_{1}\left(V_{1}\right)$ and $\widetilde{V}_{1}:=h_{3}^{-1}\left(V_{1}\right)$. Finally, let

$$
h=\Psi_{1} \circ h_{3} .
$$

Using (3-23) we obtain that

$$
F \circ h^{-1}(a, b, \vec{u})=b+\frac{1}{2} .
$$

Let $\theta>\delta$ be sufficiently close to $\delta$ satisfying the inclusion

$$
D_{1}:=[\delta, \theta] \times(-\theta, \theta) \times(-\theta, \theta)^{n-1} \subset C_{1} .
$$

Let $\widetilde{D}_{1}=h^{-1}\left(D_{1}\right) \subset \widetilde{V}_{1}$; see Figure 15 .

Lemma 3-25 If $\theta$ and $\delta$ are small enough, there is an closed ball $\tilde{W}$ in $\Omega$, containing $\widetilde{D}_{1}$, such that $h$ extends to a diffeomorphism between $\widetilde{W}$ and $[\delta, 2 /(3 \sqrt{3})] \times[-\theta, \theta] \times$ $[-\theta, \theta]^{n-1}$ with $F \circ h^{-1}(a, b, \vec{u})=b+\frac{1}{2}$, sending points with $a=2 /(3 \sqrt{3})$ to $Y$.

In the proof we shall use the following result.

Lemma 3-26 There exists a smooth curve $\gamma:[\delta, 2 /(3 \sqrt{3})] \rightarrow \Omega$, such that:

- $\gamma(2 /(3 \sqrt{3})) \in Y$.

- $\gamma(t) \in \Sigma_{1 / 2}$.

- $\gamma(t) \in \widetilde{D}_{1}$ if and only if $t \in[\delta, \theta]$.

- $h(\gamma(t))=(t, 0, \ldots, 0)$.

- $\gamma$ omits $\tilde{V} \backslash \tilde{V}_{1}$.

- $\gamma$ is transverse to $Y$.

Proof of Lemma 3-26 Let $p=h^{-1}(\theta, 0, \ldots, 0) \in \Sigma_{1 / 2}$. Let $B \subset \Sigma_{1 / 2}$ be an open ball with center $z$ and $p \in \partial B$. Let $\Sigma^{\prime}$ be the connected component of $\Sigma_{1 / 2}$ containing $p$. We consider two cases.

Case 1 If $\Sigma^{\prime} \backslash B$ is connected, it is also path connected. By (3-2), there exists a path $\tilde{\gamma} \subset \Sigma^{\prime} \backslash B$ joining $p$ with a point on the boundary. We can assume that $\tilde{\gamma}$ is transverse to $Y$. We choose $\gamma=h^{-1}([\delta, \theta] \times\{0, \ldots, 0\}) \cup \tilde{\gamma}$ (and we smooth a possible corner at $p$ ). It is clear that $\gamma$ omits $\tilde{V} \backslash \tilde{V}_{1}$ and that we can find a parametrization of $\gamma$ by the interval $[\delta, 2 /(3 \sqrt{3})]$. 


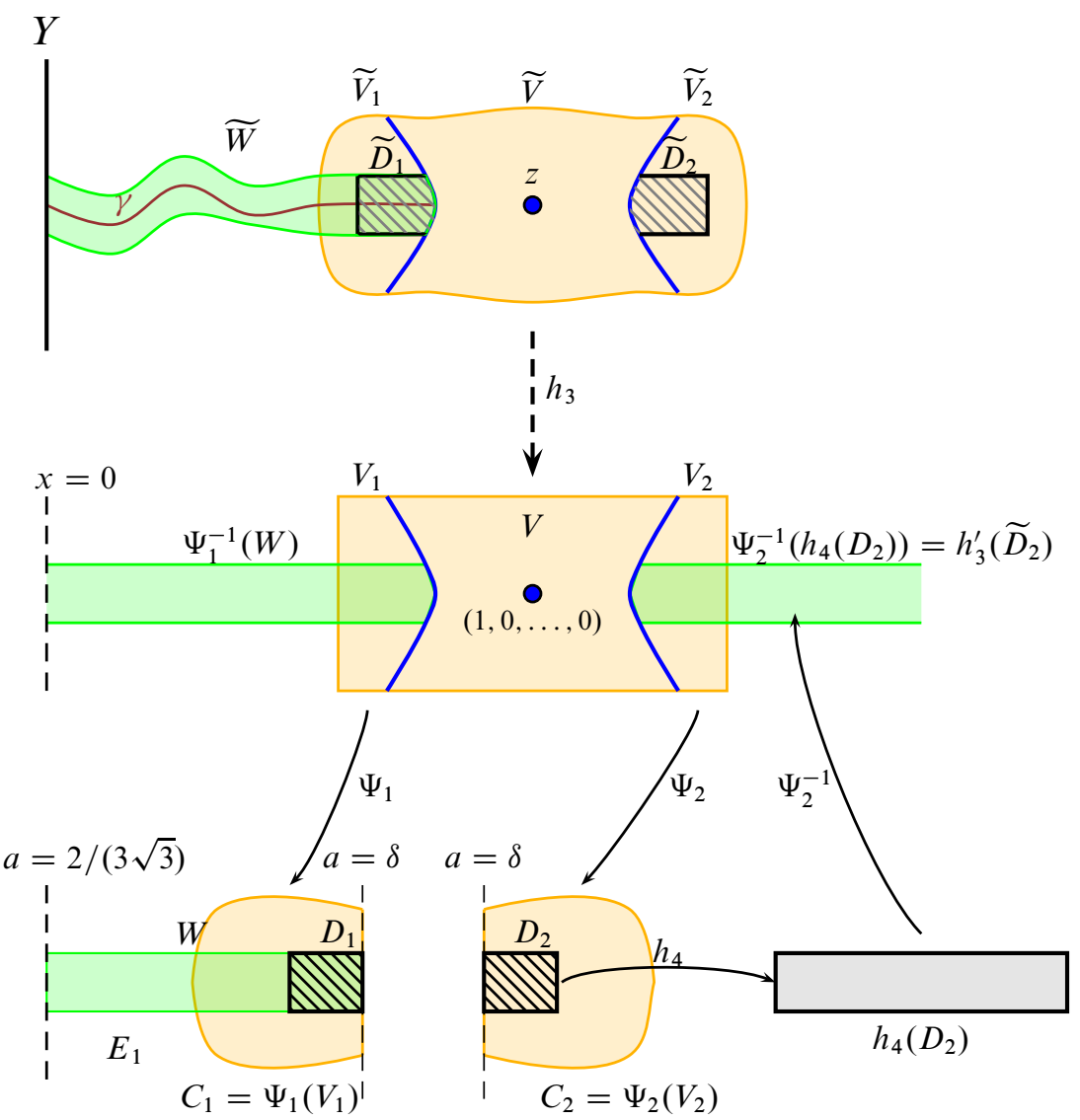

Figure 15: Notation used in Section 3.4. The top line is the picture on $\Omega$, the middle line is in coordinates such that $F$ is equal to $y^{3}-y x^{2}+y+\frac{1}{2}+\vec{u}^{2}$. The bottom line is in coordinates such that $F=b+\frac{1}{2}$. There is no mistake, the line $a=\delta$ appears twice on the picture, in coordinates on $C_{1}$ and on $C_{2}$.

Case 2 If $\Sigma^{\prime} \backslash B$ is not connected, then as $\Sigma^{\prime}$ is connected, by a homological argument we have $n=1$ and $k=1$. Since $\Sigma^{\prime}$ is connected and has boundary, then $\Sigma^{\prime}$ is an interval and $B$ is an interval too. Then $\Sigma^{\prime} \backslash B$ consists of two intervals, each intersecting $Y$. One of these intervals contains $p$. So $p$ is connected to $Y$ by an interval, which omits $B$. We conclude the proof by the same argument as in the above case, when $\Sigma^{\prime} \backslash B$ was connected.

Proof of Lemma 3-25 Given Lemma 3-26, let us choose a tubular neighborhood $X$ of $\gamma$ in $F^{-1}\left(\frac{1}{2}\right) \backslash\left(\tilde{V} \backslash \tilde{V}_{1}\right)$. Shrinking $X$ if needed we can assume that it is a disk and $X_{1}:=X \cap \widetilde{V}=\widetilde{D}_{1} \cap F^{-1}\left(\frac{1}{2}\right)$. Now let $\tau$ be the vector field on $\widetilde{D}_{1}$ given 


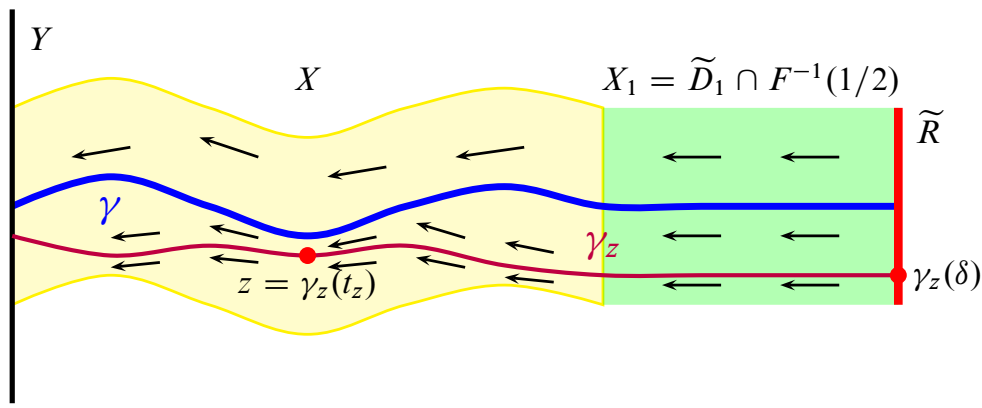

Figure 16: Proof of Lemma 3-25. Construction of the vector field $\tau$. Picture on $F^{-1}\left(\frac{1}{2}\right)$. The parallel vector field from the region on the right is extended to the whole $X$ so that it is tangent to $\gamma$.

by $(D h)^{-1}(1,0, \ldots, 0)$, where $D h$ denotes the derivative of $h$. This vector field is everywhere tangent to $X_{1}$ and

$$
\left.\tau\right|_{\gamma \cap \tilde{D}_{1}}=\frac{d}{d t} \gamma(t)
$$

by definition of $\gamma$. We extend $\tau$ to a smooth vector field on the of whole $X$, such that (3-27) holds on the whole of $\gamma$. For any point $z \in \gamma$, the trajectory of $\tau$ (which is $\gamma$ ) eventually hits $Y$ and, on the other end, it hits the "right wall"

$$
\widetilde{R}=h^{-1}\left(\{\delta\} \times\{0\} \times(-\theta, \theta)^{n-1}\right) .
$$

(compare Figure 16; note that the horizontal coordinate there increases from right to left for consistency with Figure 15). Since $\gamma$ is transverse to $\widetilde{R}$ and to $Y$, by the implicit function theorem trajectories close to $\tau$ also start at $\widetilde{R}$ and end up at $Y$. Shrinking $X$ if necessary we may assume that each point of $X$ lies on the trajectory of $\tau$ which connects a point of $\widetilde{R}$ to some point of $Y$, and all the trajectories are transverse to both $Y$ and $\widetilde{R}$.

We can now rescale $\tau$ (that is multiply by a suitable smooth function constant on trajectories) so that all the trajectories go from $\widetilde{R}$ to $Y$ in time $2 /(3 \sqrt{3})-\delta$, ie the same time as $\gamma$ does. The rescaled vector field allows us to introduce coordinates on $X$ in the following way. For any point $z \in X$, let $\gamma_{z}$ be the trajectory of $\tau$, going through $z$. We can assume that $\gamma_{z}(\delta) \in \widetilde{R}$. Let $t_{z}=\gamma_{z}^{-1}(z)$, ie the moment when $\gamma_{z}$ passes through $z$. Since we normalized $\gamma_{z}$, we know that $t_{z} \in[\delta, 2 /(3 \sqrt{3})]$ and $t_{z}=2 /(3 \sqrt{3})$ if and only if $z \in Y \cap X$. 
Let $\vec{u}_{z}$ be such that $h\left(\gamma_{z}(\delta)\right)=\left(\delta, 0, \vec{u}_{z}\right)$. The vector $\vec{u}_{z}$ might be thought of as a coordinate on $\widetilde{R}$. We define now

$$
h(z)=\left(t_{z}, 0, \vec{u}_{z}\right) .
$$

This maps clearly extends $h$ to the whole of $X$.

Now let $\tilde{W}$ be a tubular neighborhood of $X$ in $\Omega \backslash\left(\tilde{V} \backslash \tilde{V}_{1}\right)$. We use the flow of $\nabla F$ to extend coordinates from $X$ to $\tilde{W}$. More precisely, shrinking $\tilde{W}$ if needed we may assume that for each $w \in \tilde{W}$ the trajectory of $\nabla F$ intersects $X$. This intersection is necessarily transverse and it is in one point, which we denote by $z_{w} \in X$. We define now

$$
h(w)=\left(t_{z_{w}}, F(w)-F\left(z_{w}\right), \vec{u}_{z_{w}}\right) .
$$

As $h$ is a local diffeomorphism on $X$ (because $\nabla F$ is transverse to $X$ ), it is also a local diffeomorphism near $X$. We put $W=h(\tilde{W})$. Clearly both definitions of $h$ on $\tilde{V}$ and $\tilde{W}$ agree. We may now decrease $\theta$ and shrink $W$ so that

$$
W=[\delta, 2 /(3 \sqrt{3})] \times(-\theta, \theta) \times(-\theta, \theta)^{n-1} .
$$

We have $F \circ h^{-1}(a, b, \vec{u})=b+\frac{1}{2}$. We now extend $h_{3}$ over $\tilde{W}$ by the formula $h_{3}=\Psi_{1}^{-1} \circ h$.

Consider now

$$
V_{2}:=V \cap\{x>1, A(x, y) \geq \delta\} .
$$

Let $\Psi_{2}: V \rightarrow \mathbb{R}^{n+1}$ be given by $\Psi_{2}(x, y, \vec{u})=(a, b, \vec{u})=\left(A(x, y), B(x, y)+\vec{u}^{2}, \vec{u}\right)$, provided by the same formula as $\Psi_{1}$ in (3-24) but the image now satisfies $a \geqslant \delta$, cf Lemma 3-21.

Let $C_{2}=\Psi_{2}\left(V_{2}\right)$, and let us choose $\theta^{\prime}$ sufficiently small such that

$$
D_{2}:=\left[\delta, \theta^{\prime}\right] \times\left(-\theta^{\prime}, \theta^{\prime}\right) \times\left(-\theta^{\prime}, \theta^{\prime}\right)^{n-1} \subset C_{2} .
$$

We shall denote $h=\Psi_{2} \circ h_{3}$ and $\widetilde{D}_{2}=h^{-1}\left(D_{2}\right)$.

Let us now fix $M>0$ large enough and consider a map $h_{4}: \mathbb{R}^{n+1} \rightarrow \mathbb{R}^{n+1}$ of the form

$$
h_{4}(a, b, \vec{u})=(\phi(a), b, \vec{u}),
$$

where $\phi:\left[\delta, \theta^{\prime}\right] \cong[\delta, M]$ is a strictly increasing smooth function, which is an identity near $\delta$. Consider the map $h_{3}^{\prime}: \Psi_{2}^{-1} \circ h_{4} \circ h: \widetilde{D}_{2} \rightarrow \mathbb{R}^{n+1}$. Since $h$ is an identity for $a$ close to $\delta$, this map agrees with $h_{3}$ for $a$ close to $\delta$. Furthermore $F \circ h_{4}^{-1}(a, b, \vec{u})=$ $F \circ h^{-1} \circ h_{4}^{-1}(a, b, \vec{u})=b+\frac{1}{2}$ by a straightforward computation. On the other hand, 
the point $h^{-1}\left(\theta^{\prime}, 0, \ldots, 0\right) \in \widetilde{D}_{2}$ is mapped by $h_{3}^{\prime}$ to $(M, 0, \ldots, 0) \in \mathbb{R}^{n+1}$, where $M$ can be arbitrary large, eg $M>3$.

Having gathered all the necessary maps, we now conclude the proof. Let

$$
\tilde{U}=\tilde{W} \cup\left(\tilde{V} \backslash h_{3}^{-1}\left(V_{1} \cup V_{2}\right) \cup \tilde{D}_{2} .\right.
$$

The map $h_{3}: \tilde{U} \rightarrow[0, \infty) \times \mathbb{R}^{n}$ is given by $h_{3}$ on $\tilde{W}$ and on $\tilde{V} \backslash h_{3}^{-1}\left(V_{2}\right)$, and by $h_{3}^{\prime}$ on $\widetilde{D}_{2}$. This map is a diffeomorphism onto its image, so it is a chart near $z$. By construction $F \circ h_{3}^{-1}$ is equal to $y^{3}-y x^{2}+y+\vec{u}^{2}+\frac{1}{2}$ and $h_{3}(\tilde{W})$ contains the segment with endpoints $(0,0, \ldots, 0)$ and $(3,0, \ldots, 0)$. Since it is an open subset, it contains $[0,3+\eta) \times(-\eta, \eta) \times(-\eta, \eta)^{n-1}$ for $\eta>0$ small enough. The inverse image of this cube gives the required chart.

This ends the proof of Theorem 3-1 which moves a single interior critical point to the boundary. Section 4 generalizes this fact for multiple points; one of the needed tools will be the rearrangements of the critical values/points.

\subsection{Condition (3-2) revisited}

We will provide two sufficient conditions which imply Condition (3-2). One is valid for arbitrary $n \geqslant 1$, the other one holds only in the case $n=1$. We shall keep the notation from previous subsections, in particular $(\Omega, Y)$ is a cobordism between $\left(\Sigma_{0}, M_{0}\right)$ and $\left(\Sigma_{1}, M_{1}\right), F: \Omega \rightarrow[0,1]$ is a Morse function with a single critical point $z$ in the interior of $\Omega$, and $F(z)=\frac{1}{2}$. Let $\Sigma_{1 / 2}=F^{-1}\left(\frac{1}{2}\right)$ and $\Sigma^{\prime}$ be the connected component of $\Sigma_{1 / 2}$ such that $z \in \Sigma^{\prime}$.

Proposition 3-28 If $\Sigma_{0}, \Sigma_{1}$ and $\Omega$ have no closed connected components, then $\Sigma^{\prime} \cap Y \neq \varnothing$. In particular, in Theorem 3-1 we can assume that $\Sigma_{0}, \Sigma_{1}$ and $\Omega$ have no closed connected components instead of (3-2).

Proof Let $p=h^{-1}(\theta, 0, \ldots, 0) \in \widetilde{D}_{1} \subset \Omega$ and let $B$ be an open ball in $\Sigma^{\prime}$ near $z$, such that $p \in \partial B$. It is enough to show that $p$ can be connected to $Y$ by a path in $\Sigma_{1 / 2}$, which misses $B$ (compare Lemma 3-26).

Let us choose a Riemannian metric on $\Omega$. Let $W_{z}^{s}$ be the stable manifold of $z$ and let $T$ be the intersection of $W_{z}^{s}$ and $\Sigma_{0}$. This is a $(k-1)$-dimensional sphere. The flow of $\nabla F$ induces a diffeomorphism $\Phi: \Sigma_{1 / 2} \backslash B \cong \Sigma_{0} \backslash B_{0}$, where $B_{0}$ is a tubular neighborhood of $T$ in $\Sigma_{0}$ (here we tacitly use the fact that $\delta$ and $\theta$ are small enough); see Figure 17. Let $p_{0}=\Phi(p)$. Let $\Sigma_{0}^{\prime}$ be the connected component of $\Sigma_{0}$ which contains $B_{0}$. 
Now we will analyze several cases. Recall that $k=\operatorname{ind}_{z} F \in\{1, \ldots, n\}$. First we assume that $k<n$. Then $\Sigma_{0}^{\prime} \backslash T$ is connected, so $p_{0}$ can be connected to the boundary of $\Sigma_{0}^{\prime}$ — which is nonempty by the assumptions of the proposition - by a path $\gamma_{0}$. Now the inverse image $\Phi^{-1}\left(\gamma_{0}\right)$ is the required path.

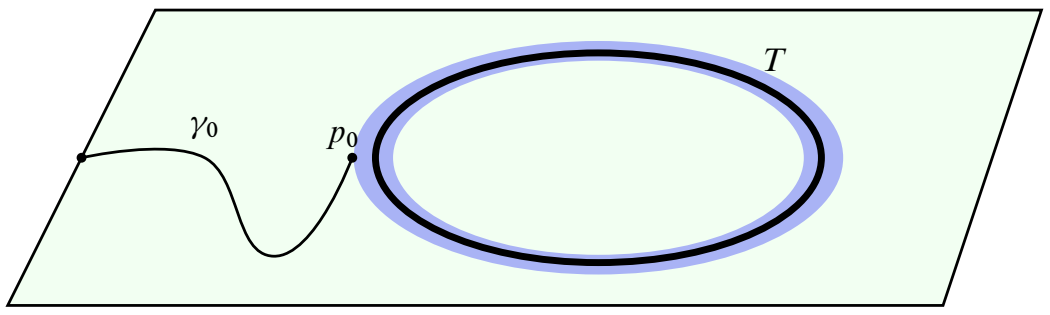

Figure 17: Notation on $\Sigma_{0}$.

If $k=n>1$ then we reverse the cobordism and look at $-F$, hence this case is covered by the previous one (since $k=n$ will be replaced by $k=1<n$ ).

Finally, it remains to deal with the situation $k=n=1$. Then $\operatorname{dim} \Sigma_{0}=1 . T$ consists of two points. Assume first that they lie in a single connected component $\Sigma_{0}^{\prime}$ of $\Sigma_{0}$. We shall show that this is impossible. As $\Sigma_{0}^{\prime}$ is connected with nontrivial boundary, it is an interval. The situation is like on Figure 18. Now as $F$ has precisely one Morse critical point of index $1, \Sigma_{1}$ is the result of a surgery on $\Sigma_{0}$. This surgery consists of removing two inner segments from $\Sigma_{0}$ and gluing back two other segments, which in Figure 18 are drawn as dashed arc. But then $\Sigma_{1}$ has a closed connected component, which contradicts assumptions of Theorem 3-1.

Therefore, $T$ lies in two connected components of $\Sigma_{0}$. The situation is drawn in Figure 19, and it is straightforward to see that $p_{0}$ (either $p_{0}^{\prime}$ or $p_{0}^{\prime \prime}$ in Figure 19) can be connected to $M_{0}$ by a segment omitting $B_{0}$.

The proof of Proposition 3-28 suggests that the case $n=1$ is different from case $n>1$. We shall provide now a full characterization of the failure to (3-2).

Proposition 3-29 Assume that $k=n=1$ and $\Omega$ is connected. If (3-2) does not hold, then $\Omega$ is a pair of pants, $\Sigma_{0}$ is a circle and $\Sigma_{1}$ is a disjoint union of two circles; or vice versa: $\Sigma_{1}=S^{1}$ and $\Sigma_{0}$ is a disjoint union of two circles. In particular, $Y=\varnothing$.

Proof A one-handle attached to a surface changes the number of boundary components by \pm 1 . Let us assume that $\Sigma_{1}$ has fewer components than $\Sigma_{0}$, if not we can reverse the cobordism. As $\Omega$ is connected, $\Sigma_{0}$ has two components and $\Sigma_{1}$ only one. Let $A_{0} \subset \Sigma_{0}$ be the attaching region, ie the union of two closed intervals to which the one-handle is 


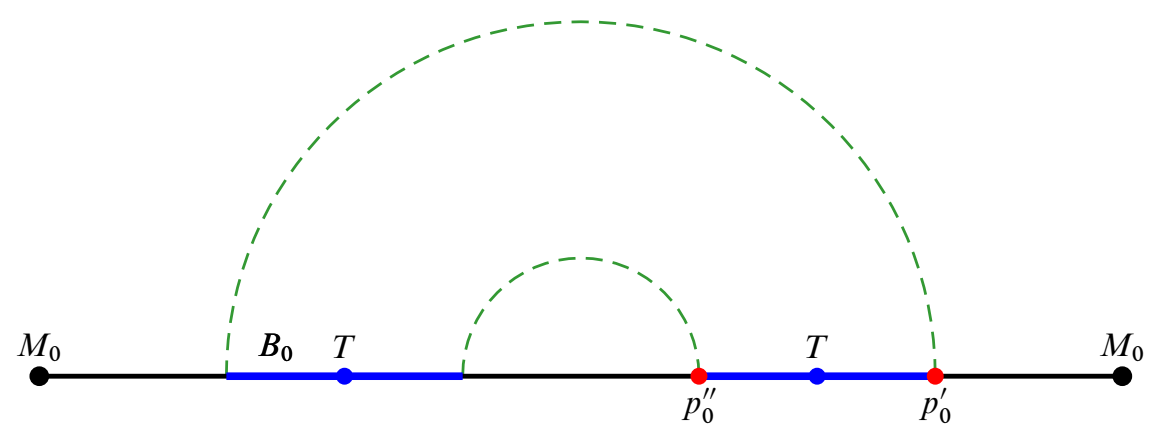

Figure 18: Proof of Proposition 3-28. Case $k=1$ and $n=1$ and $T$ lies in two components of $\Sigma . \Sigma_{0}$ is the horizontal segment. The points $p_{0}^{\prime}$ and $p_{0}^{\prime \prime}$ are the two possible positions of the point $p_{0}$.
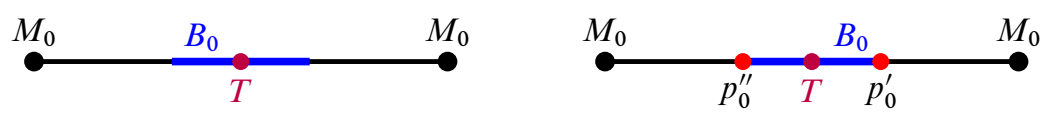

Figure 19: Proof of Proposition 3-28. Case $k=1$ and $n=1$ and $T$ lies in two components of $\Sigma_{0}$. The points $p_{0}^{\prime}$ and $p_{0}^{\prime \prime}$ are the two possible positions of the point $p_{0}$. Both can be connected to the boundary $M_{0}$.

attached. With the notation of Section 3.5 we have $\left(\Sigma^{\prime}, z\right) \cong\left(\Sigma_{0} / A_{0}, A_{0} / A_{0}\right)$, where the quotient denotes collapsing a space to a point. In particular $z$ cannot be joined to $Y$ by a path in $\Sigma^{\prime}$ if and only if $\Sigma_{0}$ is disjoint from $Y$. Hence $\Sigma_{0}$ is closed, that is, it is a union of two circles.

\section{Rearrangements of boundary handles}

\subsection{Preliminaries}

Let $(\Omega, Y)$ be a cobordism between two $n$-dimensional manifolds with boundary $\left(\Sigma_{0}, M_{0}\right)$ and $\left(\Sigma_{1}, M_{1}\right)$. Let $F$ be a Morse function, with critical points $w_{1}, \ldots, w_{k} \in$ Int $\Omega$ and $y_{1}, \ldots, y_{l} \in Y$. In the classical theory (that is, when $Y=\varnothing$ ), the ThomMilnor-Smale theorem (see [12, Section 4]) says that we can alter $F$ without introducing new critical points such that if ind $w_{i}<$ ind $w_{j}$, then $F\left(w_{i}\right)<F\left(w_{j}\right)$ as well. We want to prove similar results in our more general case.

In this section we rely very strongly on [12, Section 4$]$. 


\subsection{Elementary rearrangement theorems}

We shall begin with the case $k+l=2$, ie $F$ has two critical points. For a critical point $p$ we shall denote by $K_{p}$ the union $W_{p}^{s} \cup\{p\} \cup W_{p}^{u}$, ie the set of all points $x \in \Omega$, such that the trajectory $\phi_{t}(x)(t \in \mathbb{R})$, of the gradient vector field $\nabla F$ contains $p$ in its limit set. Elementary rearrangement theorems deal with the case when the two sets $K_{p_{1}}$ and $K_{p_{2}}$ for the two critical points are disjoint.

Proposition 4-1 (Rearrangement of critical points) Let $p_{1}$ and $p_{2}$ be two critical points, and assume that $K_{1}:=K_{p_{1}}$ and $K_{2}:=K_{p_{2}}$ are disjoint. Let us choose $a_{1}, a_{2} \in(0,1)$. Then, there exist a Morse function $G: \Omega \rightarrow[0,1]$, with critical points exactly at $p_{1}$ and $p_{2}$, such that $G\left(p_{i}\right)=a_{i}, i=1,2$; furthermore, near $p_{1}$ and $p_{2}$, the difference $F-G$ is a locally constant function.

Remark 4-2 If both critical points are on the boundary, in order to guarantee the above existence, we need even to change the Riemannian metric away from $K_{1}$ and $K_{2}$.

Proof Similarly to [12, Section 4] we will use an auxiliary result. Its proof is postponed after the end of proof of Proposition 4-1.

Lemma 4-3 There exists a smooth function $\mu: \Omega \rightarrow[0,1]$ with the following properties:

(M1) $\mu \equiv 0$ in a neighborhood of $K_{1}$.

(M2) $\mu \equiv 1$ in a neighborhood of $K_{2}$.

(M3) $\mu$ is constant on trajectories of $\nabla F$.

Furthermore, if at least one of the critical points is interior, we have

(M4) $\mu$ is constant on $Y$.

We continue with the proof of Proposition 4-1. We choose a smooth function $\Psi:[0,1] \times$ $[0,1] \rightarrow[0,1]$ with:

(PS1) $\frac{\partial \Psi}{\partial x}(x, y)>0$ for all $(x, y) \in[0,1] \times[0,1]$.

(PS2) There exists $\delta>0$ such that $\Psi(x, y)=x$ for all $x \in[0, \delta] \cup[1-\delta, 1]$ and $y \in[0,1]$.

(PS3) For any $s \in(-\delta, \delta)$ we have $\Psi\left(F\left(p_{1}\right)+s, 0\right)=a_{1}+s$ and $\Psi\left(F\left(p_{2}\right)+s, 1\right)=$ $a_{2}+s$. 
For any $\eta \in \Omega$ we define $G(\eta)=\Psi(F(\eta), \mu(\eta))$. From the properties (PS3), (M1) and (M2) we see that near $p_{i}, G$ differs from $F$ by a constant. The property (PS2) ensures that $G$ agrees with $F$ in a neighborhood of $\Sigma_{0}$ and $\Sigma_{1}$. Let us show that $\nabla G$ does not vanish away from $p_{i}$. By the chain rule we have

$$
\nabla G=\frac{\partial \Psi}{\partial x} \nabla F+\frac{\partial \Psi}{\partial y} \nabla \mu .
$$

Since $\mu$ is constant on all trajectories of $\nabla F$, the scalar product $\langle\nabla F, \nabla \mu\rangle=0$. Then the property (PS1) guarantees that $\langle\nabla G, \nabla F\rangle>0$ away from $p_{1}$ and $p_{2}$.

We need to show that $\nabla G$ is everywhere tangent to $Y$. If one of the points is interior, by (M4) $\nabla \mu$ vanishes on $Y$, hence $\nabla G$ is parallel on $Y$ to $\nabla F$ and we are done. Next assume that both critical points are on the boundary. Let us choose an open subset $U$ of $Y$ such that $\left.\nabla \mu\right|_{U}=0$ and $K_{1} \cup K_{2} \subset U$. This is possible, because of the properties (M1) and (M2). Then let us choose a neighborhood $W$ in $\Omega$ of $Y \backslash U$, disjoint from $K_{1}$ and $K_{2}$. Observe that $d G(\nabla F)=\langle\nabla G, \nabla F\rangle>0$. As $\nabla F \in T Y$ one has $T Y \not \subset$ ker $d G$, so by Lemma 1-7 we can change the metric in $W$ so that $\nabla G$ is everywhere tangent to $Y$.

Proof of Lemma 4-3 Let us define $T_{1}=K_{1} \cap \Sigma_{0}$ and $T_{2}=K_{2} \cap \Sigma_{0}$. Assume that $T_{1}$ and $T_{2}$ are not empty. For each $\eta \in \Omega \backslash K_{1} \cup K_{2}$, let $\pi(\eta)$ be the intersection of the trajectory of $\eta$ under $\nabla F$ with $\Sigma_{0}$. This gives a map $\pi: \Omega \backslash\left(K_{1} \cup K_{2}\right) \rightarrow$ $\Sigma_{0} \backslash\left(T_{1} \cup T_{2}\right)$.

Let us define $\mu$ first on $\Sigma_{0}$ by the following conditions: $\mu \equiv 1$ in a neighborhood of $T_{2}, \mu \equiv 0$ in a neighborhood of $T_{1}$. Furthermore, if either $T_{1}$ or $T_{2}$ is disjoint from the boundary $M_{0}$ we extend $\mu$ to a constant function on $M_{0}$. Finally, we extend $\mu$ to the whole $\Omega$ by picking $\mu(\eta)=\mu(\pi(\eta))$ if $\eta \notin K_{1} \cup K_{2}$, and $\left.\mu\right|_{K_{i}}(\eta)=i-1$, $i=1,2$.

If $T_{1}=\varnothing$, then ind $F p_{1}=0$ and the proof of the rearrangement theorem is completely straightforward.

\subsection{Morse-Smale condition on manifolds with boundary}

In the classical theory, the Morse-Smale condition imposed on a Morse function $F: M \rightarrow \mathbb{R}$ means that for each pair of two critical points $p_{1}, p_{2}$ of $M$ the intersection of stable manifold $W_{p_{1}}^{s}$ with the unstable manifold of $W_{p_{2}}^{u}$ is transverse. (Note that this Morse-Smale condition also depends on the choice of Riemannian metric on $M$.) Following [9, Definition 2.4.2], we reformulate the Morse-Smale condition in the following way. 
Definition 4-5 The function $F$ is called Morse-Smale if for any two critical points $p_{1}$ and $p_{2}$, the intersection of Int $\Omega \cap W_{p_{1}}^{s}$ with Int $\Omega \cap W_{p_{2}}^{u}$ is transverse (as the intersection in the $(n+1)$-dimensional manifold $\Omega)$ and the intersection of $Y \cap W_{p_{1}}^{s}$ with $Y \cap W_{p_{2}}^{u}$ is transverse (as an intersection in the $n$-dimensional manifold $Y$ ).

The Morse-Smale functions form an open-dense subset of all $C^{2}$ smooth functions satisfying the condition (1-8). The proof is the same as in the case of the Morse functions on manifolds without boundary; see for example [13, Theorem 2.27].

Assume now that $F$ is Morse-Smale. Given two critical points of $F, p_{1}$ and $p_{2}$, we want to check whether $W_{p_{1}}^{s} \cap W_{p_{2}}^{u}=\varnothing$. This depends not only on the indices, but also on whether either of the two points are boundary stable. We show this in a tabulated form in Table 1 , where ind $p_{1}=k$ and ind $p_{2}=l$. In studying the intersection, we remark that $W_{p_{1}}^{s} \cap W_{p_{2}}^{u}$ is formed from trajectories, so if for dimensional reasons we have $\operatorname{dim} W_{p_{1}}^{s} \cap W_{p_{2}}^{u}<1$, it immediately follows that this intersection is empty.

\begin{tabular}{c|c|c|c|c|c|c} 
type of $p_{1}$ & type of $p_{2}$ & $\operatorname{dim}_{\Omega} W_{p_{1}}^{s}$ & $\operatorname{dim}_{Y} W_{p_{1}}^{s}$ & $\operatorname{dim}_{\Omega} W_{p_{2}}^{u}$ & $\operatorname{dim}_{Y} W_{p_{2}}^{u}$ & empty if \\
\hline interior & interior & $k$ & $\varnothing$ & $n+1-l$ & $\varnothing$ & $k \leqslant l$ \\
interior & b. stable & $k$ & $\varnothing$ & $\varnothing$ & $n+1-l$ & always \\
interior & b. unstable & $k$ & $\varnothing$ & $n+1-l$ & $n-l$ & $k \leqslant l$ \\
\hline b. stable & interior & $k$ & $k-1$ & $n+1-l$ & $\varnothing$ & $k \leqslant l$ \\
b. stable & b. stable & $k$ & $k-1$ & $\varnothing$ & $n-l$ & $k \leqslant l$ \\
b. stable & b. unstable & $k$ & $k-1$ & $n+1-l$ & $n-l$ & $k \leqslant l$ \\
\hline b. unstable & interior & $\varnothing$ & $k$ & $n+1-l$ & $\varnothing$ & always \\
b. unstable & b. stable & $\varnothing$ & $k$ & $\varnothing$ & $n+1-l$ & $\mathbf{k}<\mathbf{l}$ \\
b. unstable & b. unstable & $\varnothing$ & $k$ & $n+1-l$ & $n-l$ & $k \leqslant l$.
\end{tabular}

Table 1: The last column shows whether there might exist trajectories from $z_{2}$ to $z_{1}$ under the Morse-Smale condition. We write $\operatorname{dim}_{\Omega} W^{s}=\operatorname{dim}\left(W^{s} \cap\right.$ Int $\Omega$ ) and $\operatorname{dim}_{Y} W^{s}=\operatorname{dim}\left(W^{s} \cap Y\right)$. $\varnothing$ means the manifold is empty.

\subsection{Global rearrangement theorem}

Let us combine the rearrangement theorems from Section 4.2 with the computations in Table 1.

Proposition 4-6 Let $F$ be a Morse function on a cobordism $(\Omega, Y)$ between $\left(\Sigma_{0}, M_{0}\right)$ and $\left(\Sigma_{1}, M_{1}\right)$. Let $w_{1}, \ldots, w_{m}$ be the interior critical points of $F$ and let $y_{1}, \ldots, y_{k+l}$ be the boundary critical points, where the points $y_{1}, \ldots, y_{k}$ are boundary stable and 
$y_{k+1}, \ldots, y_{k+l}$ are boundary unstable. Let us choose real numbers $0<c_{0}<c_{1}<$ $\cdots<c_{n+1}<1,0<c_{1}^{s}<\cdots<c_{n+1}^{s}<1,0<c_{0}^{u}<\cdots<c_{n}^{u}<1$ satisfying

$$
\begin{aligned}
& c_{i-1}^{s}<c_{i}<c_{i+1}^{s}, \\
& c_{i-1}^{u}<c_{i}<c_{i+1}^{u}, \\
& c_{i-1}^{u}<c_{i}^{s}<c_{i}^{u}
\end{aligned}
$$

for all $i \in\{0, \ldots, n+1\}$ (we can assume that $c_{-1}=c_{0}^{s}=c_{-1}^{u}=0, c_{n+2}=c_{n+2}^{s}=$ $c_{n+1}^{u}=1$ so that (4-7) makes sense for all $i$ ).

Then there exists another Morse function $G$ on the cobordism $(\Omega, Y)$ with critical points $w_{1}, \ldots, w_{m}$ in the interior, $y_{1}, \ldots, y_{k+l}$ on the boundary, such that if ind $w_{j}=$ $l$, then $G\left(w_{j}\right)=c_{l}$, if ind $y_{i}=l$ and $y_{i}$ is boundary stable then $G\left(y_{i}\right)=c_{l}^{s}$, and if ind $y_{i}=l$ and $y_{i}$ is boundary unstable, then $G\left(y_{i}\right)=c_{l}^{u}$.

Proof Given the elementary rearrangement result (Proposition 4-1), the proof is completely standard (see the proof of Theorem 4.8 in [12]). Note only that we need to have $c_{i}^{s}<c_{i}^{u}$ in the statement, because there might be a trajectory from a boundary stable critical point to a boundary unstable of the same index. However, we are free to choose $c_{i}<c_{i}^{s}$ or $c_{i} \in\left(c_{i}^{s}, c_{i}^{u}\right)$ or $c_{i}>c_{i}^{u}$.

\subsection{Moving more handles to the boundary at once}

Before we formulate Theorem 4-10, let us introduce the following technical notion.

Definition 4-8 The Morse function $F$ on the cobordism $(\Omega, Y)$ is called technically good if it has the following properties.

(TG1) If $p_{1}, p_{2}$ are (interior or boundary) critical points of $F$ then ind $p_{1}<$ ind $p_{2}$ implies $F\left(p_{1}\right)<F\left(p_{2}\right)$.

(TG2) There exist regular values of $F$, say $c, d \in[0,1]$, with $c<d$ such that $F^{-1}[0, c]$ contains those and only those critical points which have index 0 or which are boundary stable critical points of index 1 , and $F^{-1}[d, 1]$ contains those and only those critical points which have index $n+1$ and boundary unstable critical points of index $n$.

(TG3) There are no pairs of 0 and 1 (interior) handles of $F$ that can be canceled (in the sense of Section 5).

(TG4) There are no pairs of $n$ and $n+1$ (interior) handles of $F$ that can be canceled.

Lemma 4-9 Each function $F$ can be made technically good without introducing new critical points. 
Proof By Proposition 4-6 we can rearrange the critical points of $F$, proving (TG1) and (TG2). The properties (TG3) and (TG4) can be guaranteed, using the handle cancellation theorem (eg [12, Theorem 5.4]). We refer to the beginning of Section 5 for an explanation that one can use the handle cancellation theorem if the manifold in question has boundary.

Theorem 4-10 Let $(\Omega, Y)$ be a cobordism between $\left(\Sigma_{0}, M_{0}\right)$ and $\left(\Sigma_{1}, M_{1}\right)$. Let $F$ be a technically good Morse function on that cobordism, which has critical points $y_{1}, \ldots, y_{k}$ on the boundary $Y$, points $z_{1}, \ldots, z_{l+m}$ in the interior Int $\Omega$, of which $z_{m+1}, \ldots, z_{l+m}$ have index 0 or $n+1$ and the indices of $z_{1}, \ldots, z_{m}$ are in $\{1, \ldots, n\}$. Suppose furthermore the following properties are satisfied:

(I1) $\Sigma_{0}$ and $\Sigma_{1}$ have no closed connected components.

(I2) $\Omega$ has no closed connected components.

Then there exists a Morse function $G: \Omega \rightarrow[0,1]$, on the cobordism $(\Omega, Y)$, with critical points $y_{1}, \ldots, y_{k} \in Y, z_{m+1}, \ldots, z_{l+m}$ and $z_{1}^{s}, z_{1}^{u}, \ldots, z_{m}^{s}, z_{m}^{u}$ such that:

- $\operatorname{ind}_{G} y_{i}=\operatorname{ind}_{F} y_{i}$ for $i=1, \ldots, k$ and for $j=m+1, \ldots, m+l$ we have $\operatorname{ind}_{G} z_{j}=\operatorname{ind}_{F} z_{j}$.

- For $j=1, \ldots, m, \operatorname{ind}_{G} z_{j}^{s}=\operatorname{ind}_{G} z_{j}^{u}=\operatorname{ind}_{F} z_{j}$.

- For $j=1, \ldots, m, z_{j}^{s}$ and $z_{j}^{u}$ are on the boundary $Y$, furthermore $z_{j}^{s}$ is boundary stable, $z_{j}^{u}$ is boundary unstable and $G\left(z_{j}^{s}\right)<G\left(z_{j}^{u}\right)$.

In other words, we can move all critical points to the boundary at once. To prove Theorem 4-10 we use Theorem 3-1 independently for each critical point $z_{1}, \ldots, z_{m}$. We need to ensure that Condition (3-2) holds. This is done in Proposition 4-11 stated below. Given these two ingredients the proof is straightforward.

\subsection{Topological ingredients needed in the proof of Theorem 4-10}

Proposition 4-11 Let $F$ be a technically good Morse function on the cobordism $(\Omega, Y)$. Assume that $\Sigma_{0}, \Sigma_{1}$ and $\Omega$ have no closed connected components. Let $c, d$ be as in Definition 4-8.

(a) If $n>1$, then for any $y \in[c, d]$, the inverse image $F^{-1}(y)$ has no closed connected component.

(b) If $n=1$, then after possibly rearranging the critical values of the interior critical points of index 1, for any interior critical point $z \in \Omega$ of $F$ of index 1, $z$ can be connected with $Y$ by a curve lying entirely in $F^{-1}(F(z))$; and furthermore all the critical points are on different levels. 


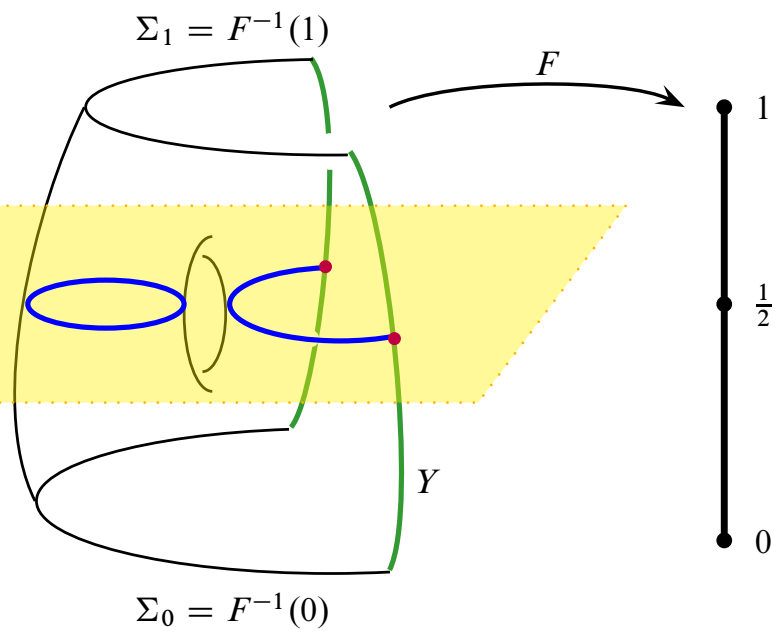

Figure 20: The statement of Proposition 4-11(a) does not hold if $n=1$. Here $F$ is the height function. The level set $F^{-1}\left(\frac{1}{2}\right)$, drawn on the picture, has two connected components, one of which is closed.

Remark 4-12 The distinction between the cases $n>1$ and $n=1$ is necessary. The conclusion of point (a) of Proposition 4-11 is not necessarily valid if $n=1$; see Figure 20 for a simple counterexample.

First let us prove several lemmas, which are simple consequences of the assumptions of Proposition 4-11. We use assumptions and notation of Proposition 4-11.

Lemma 4-13 Let $x, y \in[0,1]$ with $x<y$. If $\Omega^{\prime}$ is a connected component of $F^{-1}[x, y]$ then either $\Omega^{\prime} \cap Y=\varnothing$, or for any $u \in[x, y] \cap[c, d]$ we have $F^{-1}(u) \cap$ $\Omega^{\prime} \cap Y \neq \varnothing$.

Proof Assume that for some $u \in[x, y] \cap[c, d]$ the intersection $F^{-1}(u) \cap \Omega^{\prime} \cap Y=\varnothing$ and $\Omega^{\prime} \cap Y \neq \varnothing$. Then either $\Omega^{\prime} \cap Y \cap F^{-1}[0, u]$ or $\Omega^{\prime} \cap Y \cap F^{-1}[u, 1]$ is not empty. Assume the first possibility (the other one is symmetric) and let $Y^{\prime}=\Omega^{\prime} \cap Y \cap F^{-1}[0, u]$. Let $f=\left.F\right|_{Y^{\prime}}$ be the restriction. Then $Y^{\prime}$ is compact and $f$ has a local maximum on $Y^{\prime}$. This maximum corresponds to a critical point of $f$ of index $n$, so either a boundary stable critical point of $F$ of index $n+1$, or a boundary unstable critical point of index $n$. But the corresponding critical value is smaller than $u$, so smaller than $d$, which contradicts property (TG2).

Lemma 4-14 For any $x \in[c, 1]$ the set $F^{-1}[0, x]$ cannot have a connected component disjoint from $Y$. 
Proof Assume the contrary, and let $\Omega^{\prime}$ be a connected component of $F^{-1}[0, x]$ disjoint from $Y$. Let $\Omega_{1}$ be the connected component of $\Omega$ containing $\Omega^{\prime}$. Suppose that $\Omega_{1} \cap Y=\varnothing$. Then either $\Omega_{1} \cap\left(\Sigma_{0} \cup \Sigma_{1}\right)=\varnothing$ or $\Omega_{1} \cap\left(\Sigma_{0} \cup \Sigma_{1}\right) \neq \varnothing$. In the first case $\Omega_{1}$ is a closed connected component of $\Omega$, in the second either $\Omega_{1} \cap \Sigma_{0}$, or $\Omega_{1} \cap \Sigma_{1}$ is not empty, so either $\Sigma_{0}$ or $\Sigma_{1}$ has a closed connected component. The contradiction implies that $\Omega_{1} \cap Y \neq \varnothing$.

By Lemma 4-13 we have then $F^{-1}(x) \cap \Omega_{1} \cap Y \neq \varnothing$, hence $\Omega^{\prime \prime}:=\left(F^{-1}[0, x] \cap \Omega_{1}\right) \backslash \Omega^{\prime}$ is not empty and is disjoint from $\Omega^{\prime}$. As $\Omega^{\prime}$ and $\Omega^{\prime \prime}$ both belong to $\Omega_{1}$ which is connected, there must be a critical point $z \in \Omega_{1}$ of index 1 , which joins $\Omega^{\prime}$ to $\Omega^{\prime \prime}$. We have $F(z)>x$ and $\Omega^{\prime}, \Omega^{\prime \prime}$ belong to two different connected components of $F^{-1}[0, F(z))$ and to a single connected component of $F^{-1}[0, F(z)]$. The connected component of $F^{-1}\left[0, F(z)\right.$ ) containing $\Omega^{\prime}$ has empty intersection with $Y$ (by Lemma 4-13) hence $z$ must be an interior critical point of index 1 . We also remind the reader that all critical points of $F$ on $\Omega^{\prime}$ are interior critical points, because $\Omega^{\prime} \cap Y=\varnothing$.

Let $W^{s}$ be the stable manifold of $z$ of the vector field $\nabla F$. Then $W^{s} \cap \Omega_{1}$ must be a connected curve, with nonempty intersection with $\Omega^{\prime}$. One of its boundaries is either on $\Sigma_{0} \cap \Omega^{\prime}$ or it is a critical point of $F$ in $\Omega^{\prime}$, necessarily interior and by the Morse-Smale condition, its index is 0 . In the first case, $\Sigma_{0} \cap \Omega^{\prime}$ is not empty and since it is disjoint from $Y, \Sigma_{0}$ has a closed connected component. In the other case, we have in $\Omega_{1}$ a single trajectory between a critical point of index 0 and a critical point of index 1. This contradicts (TG3).

Lemma 4-15 Assume that for some $y \in[c, d] \Sigma_{1}$ and $\Sigma_{2}$ are two disjoint connected components of $F^{-1}(y)$. If $F$ has no interior or boundary unstable critical points of index $n$ with critical value in $[c, y)$, then $\Sigma_{1}$ and $\Sigma_{2}$ belong to two different connected components of $F^{-1}[0, y]$.

Proof For $x<y$ and close to $y$ the sets $\Sigma_{1}$ and $\Sigma_{2}$ lie in two different connected components of $F^{-1}(x, y]$. Let $x_{0} \geqslant 0$ be the smallest $x$ with that property. Assume $x_{0}>0$. Then $x_{0}$ is a critical value of $F$. The number of connected components of $F^{-1}(x)$ increases as $x$ crosses $x_{0}$. Thus the corresponding critical point is either an interior critical point of index $n$, or a boundary unstable critical point of index $n$. But then $x_{0} \geqslant c$ because of (TG2), so we have $x_{0} \in[c, y]$ which contradicts the assumptions of the lemma.

It follows that $x_{0}=0$. As $F$ has no critical points on $\Sigma_{0}$, it follows that $\Sigma_{1}$ and $\Sigma_{2}$ belong to different components of $F^{-1}[0, y]$. 
Lemma 4-16 Let $y \in[c, d]$ be chosen so that there are no interior or boundary unstable critical points of index $n$ with critical values in $[c, y)$. Then $F^{-1}(y)$ has no closed connected components.

Proof Assume that $\Sigma^{\prime}$ is a closed connected component of $F^{-1}(y)$. Let $\Sigma^{\prime \prime}=$ $F^{-1}(y) \backslash \Sigma^{\prime}$, it is not empty by Lemma 4-13 (applied for $x=0, y=1$ ), for otherwise $\Omega \cap Y=\varnothing$. Let $\Omega^{\prime}$ be the connected component of $F^{-1}[0, y]$ containing $\Sigma^{\prime}$. By Lemma 4-15, $\Omega^{\prime}$ and $\Sigma^{\prime \prime}$ are disjoint, in particular $\Omega^{\prime} \cap F^{-1}(y) \cap Y=\Sigma^{\prime} \cap Y=\varnothing$. By Lemma 4-13, $\Omega^{\prime} \cap Y=\varnothing$. But this contradicts Lemma 4-14.

Remark 4-17 There exists a symmetric formulation of the last three lemmas, which can be obtained by considering the function $1-F$ instead of $F$. For instance, in Lemma 4-16, the symmetric assumption is that there are no interior or boundary stable critical points of index 1 in $(y, d]$. The statement is the same.

Proof of Proposition 4-11 Case $n>1$ Let $x \in[c, d]$ be a noncritical value such that all the critical points of $F$ with index $n$ have critical value greater than $x$, and all critical points with index smaller than $n$ have critical values smaller than $x$. Such $x$ exists because of (TG1). If $y \leqslant x$, then Lemma 4-16 guarantees that $F^{-1}(y)$ has no closed connected components. If $y>x$, then $F^{-1}[y, d]$ has no critical points of index 1 (as $n>1$ ), so we apply the symmetric counterpart of Lemma 4-16.

Case $n=1$ The property (TG2) implies that the only critical points of $\left.F\right|_{[c, d]}$ are the interior critical points of index 1 . Let us call them $z_{1}, \ldots, z_{m}$. Since they are all of the same index, by Proposition 4-6 we are able to rearrange the values $F\left(z_{1}\right), \ldots, F\left(z_{m}\right)$ at will. Let us fix $c_{1}, \ldots, c_{m}$ with the property that $c<c_{1}<\cdots<c_{m}<d$. Let us first rearrange the points $z_{1}, \ldots, z_{m}$ so that $F\left(z_{1}\right)=\cdots=F\left(z_{m}\right)=c_{1}$.

The singular level set $F^{-1}\left(c_{1}\right)$ is a singular manifold with $m$ singular points $z_{1}, \ldots, z_{m}$, which are double points. By Lemma $4-16, F^{-1}\left(c_{1}\right)$ has no closed connected components. In particular each of the points $z_{1}, \ldots, z_{m}$, can be connected to $Y$ by a curve lying in $F^{-1}\left(c_{1}\right)$. At least one of those points can be connected to $Y$ by a curve $\gamma$, which misses all the other critical points. We relabel the critical points so that this point is $z_{m}$. We rearrange the critical points so that $F\left(z_{m}\right)=c_{m}$ and the value $F\left(z_{1}\right)=\cdots=F\left(z_{m-1}\right)=c_{1}$. By construction, $z_{m}$ can be connected to $Y$ by a curve lying in $F^{-1}\left(c_{m}\right)$.

The procedure now is repeated, ie assume that we have already moved $z_{k+1}, \ldots, z_{m}$ to levels $c_{k+1}, \ldots, c_{m}$ respectively. Then $F^{-1}\left(c_{1}\right)$ still has no closed connected components by Lemma 4-16. We assume that $z_{k}$ can be connected to $Y$ by a curve in $F^{-1}\left(c_{1}\right)$ omitting all the other critical points. Then we rearrange the critical values so that $F\left(z_{k}\right)=c_{k}$. The proof is accomplished by an inductive argument. 


\subsection{Splitting of cobordisms}

We have now all the ingredients needed to prove our main theorem about splitting cobordisms. We slightly change the notation in this subsection; the cobordism will be between $(\Sigma, M)$ and $\left(\Sigma^{\prime}, M^{\prime}\right)$.

Theorem 4-18 Let $(\Omega, Y)$ be a cobordism between $(\Sigma, M)$ and $\left(\Sigma^{\prime}, M^{\prime}\right)$. If

- $\Sigma$ and $\Sigma^{\prime}$ have no closed connected components,

- $\Omega$ has no closed connected component,

then the relative cobordism can be expressed as a union

$$
\Omega=\Omega_{0} \cup \Omega_{1 / 2} \cup \Omega_{1} \cup \Omega_{3 / 2} \cup \cdots \cup \Omega_{n+1 / 2} \cup \Omega_{n+1}
$$

such that $\partial \Omega_{s}=\Sigma_{s} \cup \Sigma_{s+1 / 2} \cup Y_{s}$ with $\Sigma_{0}=\Sigma, \Sigma_{n+3 / 2}=\Sigma^{\prime}, Y=Y_{0} \cup \cdots \cup Y_{n+1}$. In other words $\left(\Omega_{s}, Y_{s}\right)$ is a cobordism between $\left(\Sigma_{s}, M_{s}\right)$ and $\left(\Sigma_{s+1 / 2}, M_{s+1 / 2}\right)$, where $M_{s}=\partial \Sigma_{s}=\Sigma_{s} \cap Y_{s}$. Furthermore:

- $\left(\Omega_{0}, Y_{0}\right)$ is a cobordism given by a sequence of index-0 handle attachments.

- For $k=1, \ldots, n+1,\left(\Omega_{k-1 / 2}, Y_{k-1 / 2}\right)$ is a left product cobordism, given by a sequence of index- $k$ left half-handle attachments.

- For $k=1, \ldots, n,\left(\Omega_{k}, Y_{k}\right)$ is a right product cobordism, given by a sequence of index- $k$ right half-handle attachments.

- $\left(\Omega_{n+1}, Y_{n+1}\right)$ is a cobordism provided by a sequence of index- $(n+1)$ handle attachments.

Proof Let us begin with a Morse function $F$ on the cobordism which has only boundary stable critical points (see Remark 2-3). Assume that $w_{1}, \ldots, w_{m}$ are the interior critical points and $y_{1}, \ldots, y_{k}$ are the boundary critical points. After a rearrangement of critical points and the cancellation of pairs of critical points as in Lemma 4-9 we can make $F$ technically good. After applying Theorem 4-10 we get that $F$ can have only 0 handles and $n+1$ handles as interior handles. Let us write $\theta=1 /(4 n+6)$ and choose $c_{0}=\theta, c_{1}^{s}=3 \theta, c_{1}^{u}=5 \theta, \ldots, c_{k}^{s}=(4 k-1) \theta, c_{k}^{u}=(4 k+1) \theta, \ldots$, $c_{n+1}^{s}=1-3 \theta, c_{n+1}=1-\theta$. We rearrange the function $F$ according to Proposition 4-6. Then we define for $k=0, \frac{1}{2}, 1, \ldots, n+1$ the manifold $\Omega_{k}=F^{-1}[4 k \theta,(4 k+2) \theta]$, $Y_{k}=\Omega_{k} \cap Y$ and $\Sigma_{k}=F^{-1}(4 k \theta)$.

By construction, each part $\left(\Omega_{k}, Y_{k}\right)$ contains critical points only of one type: for $k=0$ and $n+1$ they are interior critical points, for $k=1, \ldots, n$ they are boundary unstable of index $k$ and for $k=\frac{1}{2}, \ldots, n+\frac{1}{2}$, they are boundary stable of index $k+\frac{1}{2}$ and we conclude the proof by Proposition 2-38. 
Remark 4-19 If the cobordism is a product on the boundary, ie $Y=M \times[0,1]$, we can choose the initial Morse function to have no critical points on the boundary. Then all the critical points of $F$ come in pairs, $z_{j}^{s}$ and $z_{j}^{u}$ with $z_{j}^{s}$ boundary stable, $z_{j}^{u}$ boundary unstable and there is a single trajectory of $\nabla F$ going from $z_{j}^{s}$ do $z_{j}^{u}$.

The strength of Theorem 4-18 is that it is much easier to study the difference between the intersection forms on $\left(\Sigma_{k}, M_{k}\right)$ and on $\left(\Sigma_{k \pm 1 / 2}, M_{k \pm 1 / 2}\right)$. We refer to [3] for an application of this fact.

\section{The cancellation of boundary handles}

In this section we assume that $F$ is a Morse function on the cobordism $(\Omega, Y)$ satisfying the Kronheimer-Mrowka-Morse-Smale regularity condition (Definition 4-5). We assume that $F$ has precisely two critical points $z$ and $w$, with ind $z=k$ and ind $w=$ $k+1$ and that there exists a single trajectory $\gamma$ of $\nabla F$ going from $z$ to $w$. If $z$ and $w$ are both interior critical points, then [12, Theorem 5.4] implies that $(\Omega, Y)$ is a product cobordism. In fact, Milnor's proof modifies $F$ only in a small neighborhood of $\gamma$, which avoids the boundary $Y$. Hence it does not matter that in our case the cobordism has a boundary.

We want to extend this result to the case of boundary critical points. In some cases an analogue of the Milnor's theorem holds, in other cases we can show that it cannot hold.

\subsection{Elementary cancellation theorems}

The following generalization of Milnor's theorem was first obtained in [7, Theorem 1].

Theorem 5-1 Let $z$ and $w$ be a boundary critical points of index $k$ and $k+1$, respectively. Assume that $\gamma$ is a single trajectory joining $z$ and $w$. Furthermore, assume that both $z$ and $w$ are boundary stable, or both boundary unstable. Then $(\Omega, Y)$ is a product cobordism.

As usual, it is enough to prove the result for boundary unstable critical points, the other case is covered if we change $F$ to $1-F$. Note also, see Section 5.2, the assumption that both critical points are boundary stable, or both boundary unstable is essential.

A careful reading of Milnor [12, pages 46-66] shows that the proof there applies to this situation with only small modifications. Below we present only three steps of that proof, adjusted to our situation. We refer to [12] for all the missing details.

Let $\xi$ be the gradient vector field of $F$. The proof relies on the following proposition (see the Preliminary hypothesis 5.5 in [12], proved on pages 55-66). 
Proposition 5-2 There exist an open neighborhood $U$ of $\gamma$ and a coordinate map $g: U \rightarrow \mathbb{R}_{\geqslant 0} \times \mathbb{R}^{n}$ and a gradient-like vector field $\xi^{\prime}$ agreeing with $\xi$ away from $U$ such that:

- $g(Y) \subset\left\{x_{1}=0\right\}$, and $g(U) \subset\left\{x_{1} \geqslant 0\right\}$.

- $g(z)=(0,0, \ldots, 0)$.

- $g(w)=(0,1, \ldots, 0)$.

- $g_{*} \xi^{\prime}=\eta=\left(x_{1}, v\left(x_{2}\right),-x_{3}, \ldots,-x_{k}, x_{k+1}, \ldots, x_{n+1}\right)$, where $v$ is a smooth function positive in $(0,1)$, zero at 0 and 1 and negative elsewhere. Moreover $\left|\frac{d v}{d x_{2}}\right|=1$ near 0 and 1 .

Furthermore, $U$ can be made arbitrary small (around $\gamma$ ).

Given the proposition, we argue in the same way as in the classical case, cf [12, pages 50-55]: we improve the vector field $\xi^{\prime}$ in $U$ so that it becomes a gradient like vector field of a function $F^{\prime}$, which has no critical points at all. Then the cobordism is a product cobordism.

The proof of Proposition 5-2 is a natural modification of Milnor's proof. After applying arguments as in [12, pages 55-58] the proof boils down to the following result.

Proposition 5-3 (cf [12, Theorem 5.6]) Let $a+b=n, a \geq 1$ and $b \geq 0$ and write a point $x \in \mathbb{R}_{\geqslant 0} \times \mathbb{R}^{a-1} \times \mathbb{R}^{b}$ as $\left(x_{a}, x_{b}\right)$ with $x_{a} \in \mathbb{R}_{\geqslant 0} \times \mathbb{R}^{a-1}$ and $x_{b} \in \mathbb{R}^{b}$. Assume that $h:\left(\mathbb{R}_{\geqslant 0} \times \mathbb{R}^{n-1},\{0\} \times \mathbb{R}^{n-1}\right) \rightarrow\left(\mathbb{R}_{\geqslant 0} \times \mathbb{R}^{n-1},\{0\} \times \mathbb{R}^{n-1}\right)$ is an orientation preserving diffeomorphism such that $h(0)=0$. Suppose that $h\left(\mathbb{R}_{\geqslant 0} \times \mathbb{R}^{a-1} \times\{0\}\right)$ intersects $\{0\} \times\{0\} \times \mathbb{R}^{b}$ only at the origin and the intersection is transverse and the intersection index is +1 . Then, given any neighborhood $N$ of $0 \in \mathbb{R}_{\geqslant 0} \times \mathbb{R}^{n-1}$, there exists a smooth isotopy $h_{t}^{\prime}$ for $t \in[0,1]$ of diffeomorphisms from $\left(\mathbb{R}_{\geqslant 0} \times \mathbb{R}^{n-1},\{0\} \times\right.$ $\mathbb{R}^{n-1}$ ) to itself with $h_{0}^{\prime}=h$ such that:

(I) $h_{t}^{\prime}(x)=h(x)$ away from $N$.

(II) $h_{1}^{\prime}(x)=x$ in some small neighborhood $N_{1}$ of 0 such that $\bar{N}_{1} \subset N$.

(III) $h_{1}^{\prime}\left(\mathbb{R}_{\geqslant 0} \times \mathbb{R}^{a-1} \times\{0\}\right) \cap\{0\} \times\{0\} \times \mathbb{R}^{b}=\{0\} \in \mathbb{R}_{\geqslant 0} \times \mathbb{R}^{n}$.

Remark 5-4 The transversality assumption from the assumption of Proposition 5-3 is equivalent to the flow of $\xi$ being Morse-Smale.

The proof of Proposition 5-3 in Milnor's book is given on pages 59-66. We prove here only the analogue of [12, Lemma 5.7]. For all other results we refer to Milnor's book. 
Lemma 5-5 Let $h$ be as in the hypothesis of Proposition 5-3. Then there exists a smooth isotopy $h_{t}: \mathbb{R}_{\geqslant 0} \times \mathbb{R}^{n-1} \rightarrow \mathbb{R}_{\geqslant 0} \times \mathbb{R}^{n-1}$, with $h_{0}$ the identity map and $h_{1}=h$, such that for each $t$ we have $h_{t}\left(\mathbb{R}_{\geqslant 0} \times \mathbb{R}^{a-1}\right) \cap \mathbb{R}^{b}=0$.

Proof We follow the proof of [12, Lemma 5.7]. We shall construct the required isotopy in two steps. First we isotope $h$ by $h_{t}(x)=\frac{1}{t} h(t x)$. Then $h_{1}=h$ and $h_{0}$ (defined to be the limit as $t \rightarrow 0$ ) is a linear map, the derivative of $h$ at 0 . If this linear map is an identity, we are done. Otherwise $h_{0}$ is just a nondegenerate linear map and clearly it maps $\mathbb{R}_{\geqslant 0} \times \mathbb{R}^{a-1} \times \mathbb{R}^{b}$ diffeomorphically onto itself. It means that under the decomposition $\mathbb{R}^{n}=\mathbb{R} \oplus \mathbb{R}^{a-1} \oplus \mathbb{R}^{b}, h_{0}$ has the block structure

$$
h_{0}=\left(\begin{array}{ccc}
a_{11} & 0 & 0 \\
* & A & B \\
* & C & D
\end{array}\right) \text {, }
$$

where $a_{11}>0$, and stars denote unimportant terms. As $h_{0}$ is orientation-preserving, $\operatorname{det} h_{0}>0$. We can apply a homotopy of linear maps which changes the first column of $h_{0}$ to $\left(a_{11}, 0, \ldots, 0\right)$ and preserves all the other entries of $h_{0}$. We do not change the determinant and the condition $h_{0}\left(\mathbb{R}_{\geqslant 0} \oplus \mathbb{R}^{a-1}\right) \cap \mathbb{R}^{b}=0$ is preserved (it means that $a_{11} \operatorname{det} A>0$ ). Let

$$
h_{00}=\left(\begin{array}{ll}
A & B \\
C & D
\end{array}\right)
$$

We have det $h_{0}=a_{11} \operatorname{det} h_{00}$, so det $h_{00}>0$. We use the same reasoning as in Milnor's proof to find a homotopy of $h_{00}$ to the identity matrix, finishing the proof.

\subsection{Noncancellation results}

The two results below have completely obvious proofs, we state them to contrast with Theorem 5-1.

Lemma 5-6 Assume that a Morse function $F$ on the cobordism $(\Omega, Y)$ between $\left(\Sigma_{0}, M_{0}\right)$ and $\left(\Sigma_{1}, M_{1}\right)$ has two critical points $z$ and $w$. Suppose $z$ is an interior critical point and $w$ is a boundary critical point. Then $(\Omega, Y)$ is not a product cobordism.

Proof $F$ restricted to $Y$ has a single critical point, so the cobordism between $M_{0}$ and $M_{1}$ cannot be trivial.

Lemma 5-7 Suppose that $F$ has two critical points $z$ and $w$. Assume that $z$ is boundary stable and $w$ is boundary unstable. Then $(\Omega, Y)$ is not a product. 
Proof If it were a product, we would have $H_{*}\left(\Omega, \Sigma_{0}\right)=0$. We shall show that this is not the case.

If $F(z)=F(w)$, then there are no trajectories between $z$ and $w$, so by Proposition 4-1 we can ensure that $F(z)<F(w)$. So we can always assume that $F(z) \neq F(w)$. For simplicity assume that $F(z)<F(w)$. Let $c$ be a regular value such that $F(z)<c<$ $F(w)$.

By Lemma 2-19 $F^{-1}[0, c] \sim \Sigma_{0} \times[0, c]$. Then $H_{*}\left(\Omega, \Sigma_{0}\right) \cong H_{*}\left(\Omega, F^{-1}[0, c]\right)$. Now $\Omega$ arises from $F^{-1}[0, c]$ by a right half-handle addition, hence $H_{*}\left(\Omega, F^{-1}[0, c]\right) \cong$ $H_{*}(H, B)$, where $(H, B)$ is the corresponding right half-handle. But $H_{*}(H, B)$ is not trivial by Lemma 2-25 (or Lemma 2-18).

\section{References}

[1] V I Arnol' d, S M Guseĭn-Zade, A N Varchenko, Singularities of differentiable maps, I: The classification of critical points, caustics and wave fronts, Monographs in Mathematics 82, Birkhäuser, Boston, MA (1985) MR777682

[2] J M Bloom, The combinatorics of Morse theory with boundary, from: "Proceedings of the Gökova Geometry-Topology Conference 2012”, (S Akbulut, D Auroux, T Önder, editors), Int. Press, Somerville, MA (2013) 43-88 MR3203357

[3] M Borodzik, A Némethi, A Ranicki, Codimension 2 embeddings, algebraic surgery and Seifert forms, preprint (2012) arXiv:1211.5964

[4] M Borodzik, A Némethi, A Ranicki, On the semicontinuity of the mod 2 spectrum of hypersurface singularities, J. Algebraic Geom. 24 (2015) 379-398 MR3311588

[5] D Braess, Morse-Theorie für berandete Mannigfaltigkeiten, Math. Ann. 208 (1974) 133-148 MR0348790

[6] M Goresky, R MacPherson, Stratified Morse theory, Ergeb. Math. Grenzgeb. 14, Springer, Berlin (1988)

[7] B Hajduk, Minimal m-functions, Fund. Math. 111 (1981) 179-200 MR611759

[8] A Jankowski, R Rubinsztein, Functions with non-degenerate critical points on manifolds with boundary, Comment. Math. Prace Mat. 16 (1972) 99-112 MR0331409

[9] P Kronheimer, T Mrowka, Monopoles and three-manifolds, New Mathematical Monographs 10, Cambridge Univ. Press (2007) MR2388043

[10] F Laudenbach, A Morse complex on manifolds with boundary, Geom. Dedicata 153 (2011) 47-57 MR2819662

[11] J Milnor, Morse theory, Ann. Math. Studies 51, Princeton Univ. Press (1963) MR0163331 
[12] J Milnor, Lectures on the h-cobordism theorem, Princeton Univ. Press (1965) MR0190942

[13] L I Nicolaescu, An invitation to Morse theory, Springer, Berlin (2007) MR2298610

[14] A Ranicki, High-dimensional knot theory: Algebraic surgery in codimension 2, Springer, New York (1998) MR1713074

[15] D Salamon, Morse theory, the Conley index and Floer homology, Bull. London Math. Soc. 22 (1990) 113-140 MR1045282

[16] S Smale, On gradient dynamical systems, Ann. Math. 74 (1961) 199-206 MR0133139

[17] S Smale, On the structure of manifolds, Amer. J. Math. 84 (1962) 387-399 MR0153022

[18] E Witten, Supersymmetry and Morse theory, J. Differential Geom. 17 (1982) 661-692 MR683171

Institute of Mathematics, University of Warsaw

ul. Banacha 2, 02-097 Warszawa, Poland

Alfréd Rényi Institute of Mathematics

Reáltanoda u. 13-15., 1053 Budapest, Hungary

School of Mathematics, University of Edinburgh

Edinburgh EH9 3JZ, UK

mcboro@mimuw.edu.pl, nemethi.andras@renyi.mta.hu, nemethi@renyi.hu, a.ranicki@ed.ac.uk

Received: 23 March 2015 
